\title{
Quantum-improved Schwarzschild-(A)dS and Kerr-(A)dS spacetimes
}

\author{
Jan M. Pawlowski ${ }^{1,2}$ and Dennis Stock ${ }^{3,1}$ \\ ${ }^{1}$ Institut für Theoretische Physik, Universität Heidelberg, \\ Philosophenweg 16, 69120 Heidelberg, Germany \\ ${ }^{2}$ ExtreMe Matter Institute EMMI, GSI Helmholtzzentrum für Schwerionenforschung mbH, \\ Planckstr. 1, 64291 Darmstadt, Germany \\ ${ }^{3}$ University of Bremen, Center of Applied Space Technology and Microgravity (ZARM), \\ 28359 Bremen, Germany
}

(Received 21 August 2018; published 12 November 2018)

\begin{abstract}
We discuss quantum black holes in asymptotically safe quantum gravity with a scale identification based on the Kretschmann scalar. After comparing this scenario with other scale identifications, we investigate in detail the Kerr-(A)dS and Schwarzschild-(A)dS spacetimes. The global structure of these geometries is studied as well as the central curvature singularity and test particle trajectories. The existence of a Plancksized, extremal, zero-temperature black hole remnant guarantees a stable end point of the evaporation process via Hawking radiation.
\end{abstract}

DOI: $10.1103 /$ PhysRevD.98.106008

\section{INTRODUCTION}

The consistent quantization of gravity is an open challenge to date. One of the candidates is the asymptotic safety (AS) scenario for quantum gravity [1], its attraction being the possible quantum-field theoretical ultraviolet completion of the standard model with gravity. If realized, it is the minimal UV closure of high-energy physics including gravity within a purely field-theoretical setup.

One of the prominent and characteristic properties of asymptotically safe gravity is its ultraviolet scaling regime for momentum scales $k$ larger than the Planck scale $M_{\mathrm{pl}}$. In the AS setup, the latter is defined as the scale beyond which quantum gravity corrections dominate the physics and agrees well with the classical Planck scale. In this regime, the Newton's coupling $G$ and cosmological constant $\Lambda$, as well as all further couplings of terms, e.g., of the higher curvature invariants $R^{n}$, run according to their canonical scaling. For the Newton's coupling and cosmological constant in particular, this entails $G(k) \propto 1 / k^{2}$ and $\Lambda(k) \propto k^{2}$, respectively, instead of the classical constant behavior. Consequently, the physics at these scales looks rather different to that of general relativity.

Black holes offer one of the few possibilities where such deviations from classical general relativity may be observed as they feature large curvatures. Asymptotically

Published by the American Physical Society under the terms of the Creative Commons Attribution 4.0 International license. Further distribution of this work must maintain attribution to the author(s) and the published article's title, journal citation, and DOI. Funded by SCOAP ${ }^{3}$. safe quantum black holes have been amongst the first applications of asymptotically safe gravity after its first explicit realization within the functional renormalization group [2]. Within such a renormalization group setting, the Newton's coupling and cosmological constant are naturally elevated to couplings running with the momentum (RG) scale $k$. Then, classical solutions of the Einstein field equations are quantum improved by replacing Newton's and the cosmological constant by functions depending on a respective length scale. The $k$-dependent RG-runnings, equipped with an identification between momentum and length scales, serve as an ansatz for these functions. The earliest works investigated the Schwarzschild spacetime [3,4] followed by studies of the Kerr spacetime [5] and Schwarzschild-(A)dS geometries [6]. Black holes in higher dimensions have been studied in [7]. All works, summarized in [8], match the classical results of general relativity in the low energy limit, but show significant changes for the number of horizons, test particle trajectories, the Hawking temperature, and the entropy around the Planckian regime. There is evidence for a cold, extremal Planck-sized remnant, which is a smallest black hole with zero temperature, a possibly promising answer to the end point of black hole evaporation. By studying dynamical, nonvacuum solutions such as the Vaidya spacetime, the processes of black hole formation [9] and evaporation [10] can be addressed directly, leading to the same conclusions as above. The quantum effects render the central curvature singularity at $r=0$ less divergent, some scenarios lead to a complete resolution. A detailed study on the implications for the laws of black hole thermodynamics was performed in [11]. Most of the above results for a quantum-improved spacetime were obtained by using a cutoff identification 
based on a classical spacetime. This was addressed in $[12,13]$, where a consistent framework with an underlying quantum spacetime was introduced.

In this work, we present a new scale identification based on the quantum-improved classical Kretschmann scalar. This approach takes the running of the couplings into account which removes unphysical features in the resulting geometries. For the first time in this quantum gravity setup, the Kerr-(A)dS geometry, as the most general vacuum black hole solution including a cosmological constant, is studied in great detail. As a special case $(a=0)$, the results for Schwarzschild-(A)dS are presented separately. The ordinary Schwarzschild and Kerr solutions are also contained by setting the cosmological constant to zero.

This work is structured as follows: we start with a brief review of the AS scenario of quantum gravity in Sec. II, and discuss the studied geometries in Sec. III. The novel scale identification is discussed in Sec. IV. Results on horizons and the GR-limit are presented in Sec. V, the global structure in Sec. VI, test particle trajectories in Sec. VIC, the curvature singularity in Sec. VII, and Hawking temperatures and the black hole evaporation process in Sec. VIII. Some technical details are deferred to the appendices which contain in particular a discussion of proper distance matchings; see Appendix C.

\section{ASYMPTOTIC SAFE QUANTUM GRAVITY}

By now, asymptotically safe quantum gravity has been studied in an impressive wealth and depth of approximations including higher derivative terms, the full $f(R)$ potential as well as the inclusion of matter, see e.g., [14-19] and references therein. The specific shape of the running of $G(k)$ and $\Lambda(k)$ depends on the regularization scheme or regulator which also defines part of the scale identification. Moreover, despite the advances in the approximation schemes used in recent computations, the systematic error estimates are still relatively large. However, while these details do not affect the results of this work qualitatively, all runnings have to meet the following general constraints:

(1) The existence of a UV fixed point, that is, the dimensionless couplings $g$ and $\lambda$ become constant in the UV-limit:

$$
(g, \lambda) \stackrel{k \rightarrow \infty}{\longrightarrow}\left(g_{*}, \lambda_{*}\right) .
$$

(2) The effective theory should recover the classical theory of general relativity in the IR-limit, i.e., $G$ and $\Lambda$ approach Newton's constant $G_{0}$ and a cosmological constant $\Lambda_{0}$ respectively, reducing the effective action to the Einstein-Hilbert action:

$$
G, \Lambda \stackrel{k \rightarrow 0}{\longrightarrow} G_{0}, \Lambda_{0} \Leftrightarrow g \sim k^{2} \text { and } \lambda \sim k^{-2} .
$$

The running of $g(k)$ and $\lambda(k)$ is typically obtained numerically. In the following, we approximate them by analytical expressions, which show the same features and are compatible with the above constraints in the UV and IR. For instance, a comparison with the results of the systematic vertex expansion up to the fourth order in [20] is provided in Fig. 18 in the Appendix. The following scale runnings are used:

$$
\begin{aligned}
& g(k)=\frac{G_{0} g_{*} k^{2}}{g_{*}+G_{0} k^{2}} \Leftrightarrow G(k)=\frac{G_{0} g_{*}}{g_{*}+G_{0} k^{2}}, \\
& \lambda(k)=\frac{\Lambda_{0}}{k^{2}}+\lambda_{*} \Leftrightarrow \Lambda(k)=\Lambda_{0}+\lambda_{*} k^{2} .
\end{aligned}
$$

The functional dependence of $g(k)$ was already used in [4] and $\lambda(k)$ agrees with the expression used in [8] without the logarithmic term. $G_{0}$ and $\Lambda_{0}$ are the IR values of the gravitational and cosmological coupling, whereas $g_{*}$ and $\lambda_{*}$ are the fixed point values of the dimensionless couplings. In the following analysis, we choose the numerical values at the fixed point to be the ones for the background couplings obtained in Appendix B of [20], together with their identification scheme in (34):

$$
g_{*}=1.4, \quad \lambda_{*}=0.1 .
$$

The dependence of Newton's coupling $G(k)$ and cosmological constant $\Lambda(k)$ on the running scale $k$ reflects the nontrivial dependence of the full effective action at vanishing cutoff scale on the Laplacian $\Delta$, as well as the existence of higher-order terms. As in earlier works, we use the following strategy to take into account these terms: we use solutions to the Einstein field equations and assume that quantum gravity effects can be modeled by momentumdependent $G$ and $\Lambda$, equipped with a relation to convert the momentum into a length scale. The now $r$-dependent $G$ and $\Lambda$ are inserted back into the classical solution, yielding a quantum-improved spacetime. This procedure is the analogue of the Uehling's correction in QED, see [21,4] for more details. In the context of asymptotically save gravity, it has been shown in [6], that a quantum-improved metric in the above sense can be a solution to the field equations derived from the quantum-improved Einstein-Hilbert action in the UV-limit, at least in the spherically symmetric case. Furthermore, the quantum-improved metric, together with its observables, approach the results obtained from general relativity in the IR, and thus show the correct low energy limit.

In the following we need the couplings $G(r)$ and $\Lambda(r)$ as functions of radius $r$ rather than momentum scale $k$. Thus, we have to establish a relation $k(r)$ in order to arrive at $G(k(r)), \Lambda(k(r))$. A commonly used ansatz for $k(r)$ is

$$
k(r)=\frac{\xi}{D(r)},
$$

with constant $\xi$ and a $r$-dependent function $D$ with momentum dimension minus one (length), encoding the 
physical scales. Our choice $\xi=1 / \sqrt{\lambda_{*}}$ is further motivated in Appendix A.

\section{INVESTIGATED GEOMETRIES}

In this work, we study geometries based on solutions of the Einstein equations with cosmological constant, but vanishing stress-energy tensor. Depending on the sign of the cosmological constant, the spacetime is called asymptotically de Sitter (dS), flat, or anti-de Sitter (AdS). As the stress-energy tensor is zero, the black hole is allowed to have a mass and angular momentum, but no charge. Thus, we study the Schwarzschild-(A)dS spacetime of a nonrotating black hole and the Kerr-(A)dS spacetime for a rotating black hole.

The Kerr-(A)dS geometry is the most general vacuum black hole solution, which includes a cosmological constant. Hence the Schwarzschild-(A)dS as well as the Schwarzschild and Kerr solutions in flat space can be obtained from Kerr-(A)dS by either setting the rotations parameter $a$ or the cosmological coupling $\Lambda$ to zero. In our analysis, we discuss the quantum-improved Schwarzschild(A)dS and Kerr-(A)dS solution, but the results can be easily extended to asymptotically flat spacetimes. Below we briefly summarize some basic properties of these geometries.

\section{A. Schwarzschild-(A)dS}

The Schwarzschild-(A)dS solution is a two-parameter family of solutions of the nonvacuum Einstein equations, labeled by $(M, \Lambda)$. It is explicitly given by

$$
\begin{aligned}
\mathrm{d} s^{2} & =-f(r) \mathrm{d} t^{2}+f^{-1}(r) \mathrm{d} r^{2}+r^{2} \mathrm{~d} \Omega^{2}, \\
f(r) & :=1-\frac{2 M G}{r}-\frac{\Lambda}{3} r^{2},
\end{aligned}
$$

with $t \in(-\infty, \infty), r \in(0, \infty)$, Newton's constant $G$, the cosmological constant $\Lambda$, and $\mathrm{d} \Omega^{2}$ the metric on $S^{2}$. This solution is spherically symmetric and displays a curvature singularity at $r=0$ if $M \neq 0$. For $\Lambda=0$, it reduces to the Schwarzschild solution in flat space and for $M=0$ but $\Lambda \neq 0$, one obtains the metric describing AdS or $\mathrm{dS}$, depending on the sign of $\Lambda$. Therefore, this metric interpolates between a Schwarzschild solution on small scales and an (A)dS solution on large scales. Horizons are solutions to $f(r)=0$.

\section{B. Kerr-(A)dS}

The Kerr-(A)dS solutions form a three parameter family, labeled by $(M, J, \Lambda)$. Unlike in the flat case, $M$ and $J$ cannot be interpreted as mass and angular momentum of the black hole anymore; however, for convenience, we still refer to them as mass and angular momentum in the text below. The metric is given by [22],

$$
\begin{aligned}
\mathrm{d} s^{2}= & -\frac{\Delta_{r}}{\rho^{2} \Xi^{2}}\left(\mathrm{~d} t-a \sin ^{2} \theta \mathrm{d} \phi\right)^{2}+\frac{\rho^{2}}{\Delta_{r}} \mathrm{~d} r^{2}+\frac{\rho^{2}}{\Delta_{\theta}} \mathrm{d} \theta^{2} \\
& +\frac{\Delta_{\theta} \sin ^{2} \theta}{\Xi^{2} \rho^{2}}\left(a \mathrm{~d} t-\left(r^{2}+a^{2}\right) \mathrm{d} \phi\right)^{2},
\end{aligned}
$$

with

$$
\begin{aligned}
a & :=\frac{J}{M}, \\
\rho^{2} & :=r^{2}+a^{2} \cos ^{2} \theta, \\
\Delta_{r} & :=\left(r^{2}+a^{2}\right)\left(1-\frac{\Lambda}{3} r^{2}\right)-2 G M r, \\
\Delta_{\theta} & :=1+\frac{\Lambda}{3} a^{2} \cos ^{2} \theta, \\
\Xi & :=1+\frac{\Lambda}{3} a^{2} .
\end{aligned}
$$

The parameter $a$ is referred to as rotation parameter and is restricted by

$$
\frac{1}{3} \Lambda a^{2}>-1
$$

in order to preserve the Lorentzian signature of the metric. The coordinate ranges are $t \in(-\infty, \infty), r \in(0, \infty), \theta \in$ $[0, \pi]$ and $\phi \in[0,2 \pi)$. It can be shown that this solution reduces to a Kerr black hole in the limit of small $r$, whereas for large $r$ it gives back the metric of (A)dS. In the case of $a=0$, one recovers the Schwarzschild-(A)dS metric of a nonrotating black hole (6). For $\Lambda=0$, the metric reduces to the one of a Kerr black hole in flat space. For $M=0$ and $a=0$, we recover (A)dS. For $M \neq 0$, there is a curvature singularity at $r=0$ in the equatorial plane $\theta=\frac{\pi}{2}$. Horizons correspond to solutions of $\Delta_{r}=0$.

\section{SCALE IDENTIFICATION}

In pure gravity systems, i.e., systems with vanishing stress-energy tensor, there is no unique way to fix the scale identification. In fact, it turns out that physical features of the spacetime such as the number of horizons, Hawking temperatures and the strength of the curvature singularity actually do depend on the particular choice of $k(r)$. Motivated by dimensional analysis, one simple way to identify the momentum scale of the FRG setup with a length scale is an inverse proportionality. However, this ansatz is completely insensitive to typical scales of the underlying spacetime. Therefore, different scale setting procedures have been brought forward, for instance on the level of the field equations, e.g., [6]. A more feasible approach to account for spacetime features is to use proper distance integrals. As such, they give rise to diffeomorphism invariant quantities. Proper distance integrals based on classical spacetimes were suggested in [4]. Later, it was 
pointed out in $[12,13]$, that this procedure can be upgraded to a consistent setting by computing the proper distance already in the quantum-improved geometry.

Here, we investigate this approach for Schwarzschild(A)dS and Kerr-(A)dS spacetimes. However, using two different integration contours for the computation of the proper distance in the upgraded scheme yields ill-defined quantities. In the case of a radial integration path, we find diverging surface gravities for all horizons. This results in divergent Hawking temperatures, independent of the black hole parameters. In the case of a path prescribed by the timelike geodesic of an infalling observer, we find an identically vanishing eigentime. The analysis and results for the proper distances are given in Appendix C.

In light of these results, a different identification scheme is required. Such a scheme has to be based on other diffeomorphism invariant quantities, for example on curvature scalars. In cosmological contexts, the Ricci scalar $R$ has been used [23,24]. However, the classical Ricci scalar cannot be used, since it vanishes identically for vacuum solutions of the Einstein field equations. Thus, in the following analysis, we will base our scale identification on the Kretschmann scalar $K=R_{\alpha \beta \gamma \delta} R^{\alpha \beta \gamma \delta}$, a diffeomorphism invariant quantity of momentum dimension four. This motivates the scale identification

$$
D_{\mathrm{K}}(r)=\frac{1}{\chi\left(K-K_{\infty}\right)^{1 / 4}},
$$

with a constant $\chi$, chosen to be $\chi=\left(\frac{1}{8}\right)^{1 / 4}$ in the following calculations, and $K_{\infty}=K(r=\infty)=8 / 3 \Lambda_{0}^{2}$, using (11). We subtract the Kretschmann scalar at $r \rightarrow \infty$; otherwise, $D(r)$ would approach a constant in the IR and therefore $G$ and $\Lambda$ would fail to display the correct IR-limit $G_{0}$ and $\Lambda_{0}$, respectively, cf. (3). For simplicity, we base the matching on the classical Kretschmann scalar in the equatorial plane $(\theta=\pi / 2)$. For both Kerr-(A)dS and Schwarzschild-(A) $\mathrm{dS}$, we arrive at

$$
K=\frac{8}{3} \Lambda^{2}+\frac{48 M^{2}}{r^{6}} G^{2} .
$$

The quantum-improved version of the classical Kretschmann scalar (11), referred to as $K_{\text {qu }}$, provides a consistent framework accounting for typical scales of the underlying (quantum) geometry. Of course, it would be desirable to use the true Kretschmann scalar, computed directly from the quantum-improved metric. This is left for future work. On a technical level, the RG-improved version turns (10) into a functional equation for $D_{\mathrm{K}}(r)$. In order for this equation to have a positive, real solution, $\chi$ must be constrained to $\chi<(3 / 8)^{1 / 4}$, such that the expression under the root in the UV-expression in Table II remains positive. In Appendix $\mathrm{A}$, we discuss the impact of $\chi$ on the results. Also, the quantum-improved version of classical
Kretschmann scalar (11) approaches the classical version for $r \rightarrow \infty$, but this does not hold for $D_{\mathrm{K}}$, given by (10), because $K_{\mathrm{qu}} \rightarrow K_{\infty}$ is faster than $K_{\mathrm{cl}} \rightarrow K_{\infty}$. The curvature near the singularity, the construction of the Penrose diagrams, and the UV-limits for each proper distance are discussed in Appendix E.

\section{LAPSE FUNCTION AND NUMBER OF HORIZONS}

With the running couplings $G$ and $\Lambda$ from the previous section, physical properties of the quantum-improved spacetimes can be deduced. Central tools are the lapse functions $f(r)$ and $\Delta(r)$, whose roots determine the location of horizons in the spacetime. These zeros are shown to be Killing horizons in Appendix B, implying that they can be assigned a constant surface gravity, which turns out to be proportional to the first derivative of the lapse function evaluated at the horizon. This can be used to address thermodynamical processes such as the end point of black hole evaporation via Hawking radiation. Another interesting question is that of the similarity of the quantumimproved geometry to the classical geometry in general relativity, serving as a metric ansatz for the quantum improvement.

In this section, we will discuss the lapse functions $f(r)$ and $\Delta(r)$ for the Kretschmann matching by determining the number of horizons and comparing them with the lapse functions of general relativity. We first start with asymptotically AdS spacetimes, i.e., $\Lambda_{0}<0$, and comment on the results for $\Lambda_{0}>0$ subsequently. The results for all other matchings can be found in Appendix C.

\section{A. Schwarzschild-AdS}

Classically, i.e., for constant $G \& \Lambda_{0}<0$, the lapse function $f(r)$ shows just one zero corresponding to the event horizon of the black hole, whereas the quantumimproved Schwarzschild geometry shows up to two horizons, if a consistent matching is adopted; see Fig. 1. Starting at very large masses, well above the Planck mass, we find two horizons, generated by a minimum of the lapse function. Comparing with the classical lapse function in Fig. 2 shows that the outer horizon of the quantumimproved spacetime coincides with the event horizon of the classical black hole. The larger the mass, the better the agreement and the more the inner horizon moves towards zero. Hence, increasing the mass makes the black holes more classical. Decreasing the mass causes the minimum to shrink and the horizons to move towards each other. There exists a critical mass $M_{\mathrm{c}}$ around two Planck masses, $M_{\mathrm{c}} \approx 2 M_{\mathrm{Pl}}$, when the minimum is also a zero of the lapse function. Then, both horizons merge and $f(r)$ has a double root. We will see later, that this geometry is similar to a classical, extreme Reissner-Nordström black hole in AdS. 


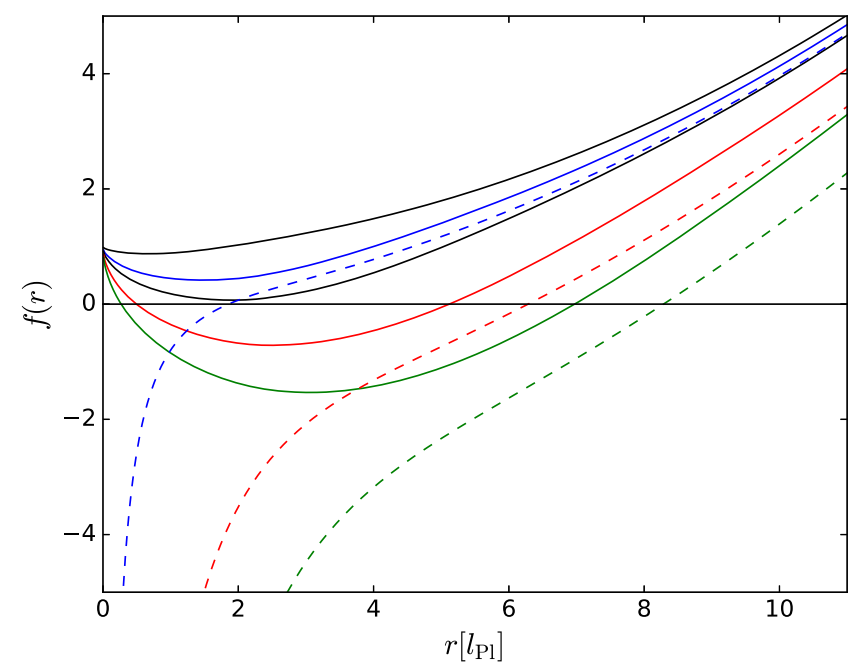

FIG. 1. $f(r)$ from (6) based on the Kretschmann scalar matching for increasing mass from top to bottom. Results based on the quantum-improved Kretschmann scalar are given by solid curves, whereas results based on the classical Kretschmann scalar are dashed. The parameters are $\Lambda_{0}=-0.1$ and $M=0.1,1,2,5,9 M_{\mathrm{Pl}}$. Curves of the same mass have the same color.

For masses below the critical mass, the minimum is above zero and no horizons are present.

The results for matchings computed in spacetimes with running couplings agree with the matchings based on spacetimes with constant couplings on the position of the outer horizon, but differ significantly for smaller radii.

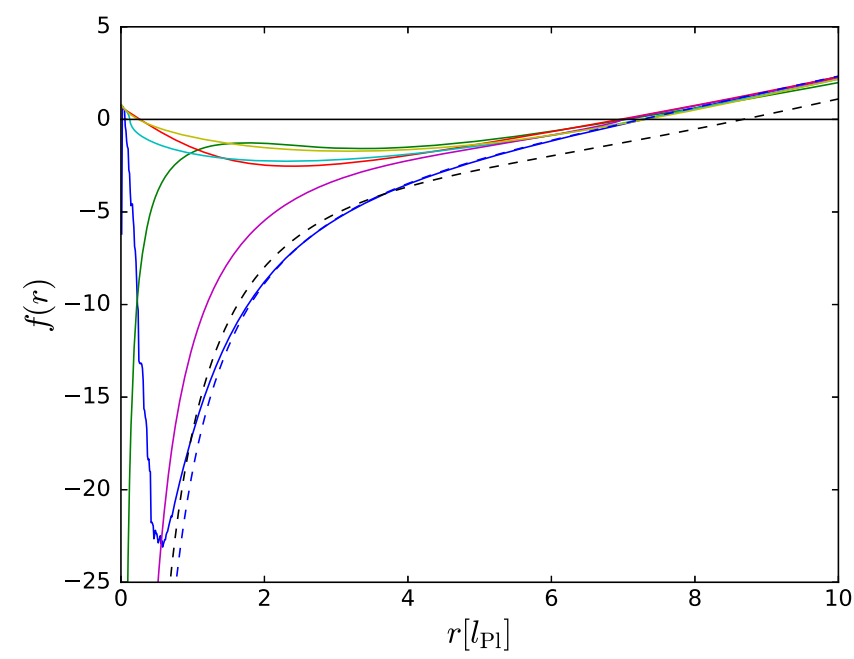

FIG. 2. Comparison of $f(r)$ for all matchings with the classical result from general relativity for $M=10 M_{\mathrm{Pl}}$ and $\Lambda_{0}=-0.1$. Matching based on the quantum geodesic in dark blue, classical geodesic in dark green, quantum radial path in light blue, classical radial path in purple, quantum Kretschmann scalar in light green, classical Kretschmann scalar in dashed black, linear matching in red and the result from general relativity in dashed dark blue. All matchings, apart from the classical Kretschmann setting, agree with the classical position of the outer black hole horizon.
These differences emerge because in the latter case, the matching is based on a classical geometry, whereas we actually study a quantum geometry with running couplings. Varying the amplitude for negative $\Lambda_{0}$ does not affect the qualitative results, but changes the scale.

\section{B. Kerr-AdS}

A classical, nonextremal Kerr-AdS spacetime has two horizons: a Cauchy horizon inside the black hole event horizon. In contrast to the Schwarzschild case discussed above, the quantum improvement of this spacetime does not allow for more horizons than in the classical geometry. Since the proper distances vanish identically in the consistent scenarios, we show only the results for the Kretschmann matching in Fig. 3 and the dependence on the rotation parameter for fixed mass in Fig. 4. The results for the linear matching can be found in Appendix C. In general, the consistent quantum-improved version displays the same behavior as the classical solution. However, the inner horizon in the quantum-improved spacetime is located at larger radii than the classical Cauchy horizon; see Fig. 5.

\section{Asymptotically de Sitter spaces}

If we take the spacetime to be asymptotically de Sitter, we find the possibility to get up to three horizons. The additional horizon is generated by the positive cosmological constant in the IR and appears in the classical regime at large radii. The typical shapes of $f(r)$ and $\Delta_{r}(r)$ are displayed in Figs. 6 and 7 for the Kretschmann matching, the dependence on the amplitude of $\Lambda_{0}$ is shown in Figs. 8

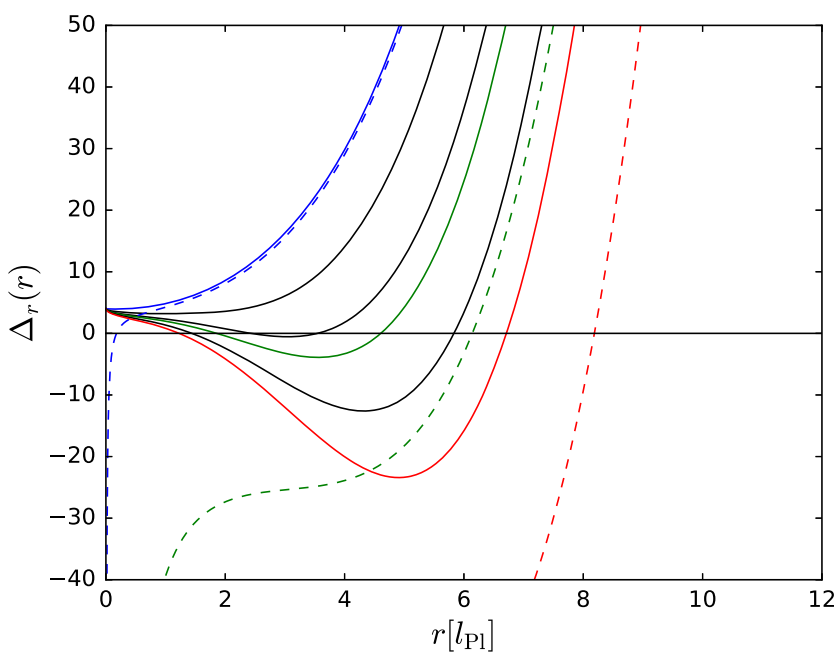

FIG. 3. $\Delta_{r}(r)$ from (8) based on the Kretschmann scalar matching for increasing mass from top to bottom. Results based on the quantum-improved Kretschmann scalar are given by solid curves, whereas results based on the classical Kretschmann scalar are dashed. With parameters $\Lambda_{0}=-0.1, a=2$ and $M=0.1,2,4,5,7,9 M_{\mathrm{Pl}}$. Curves of the same mass have the same color. 


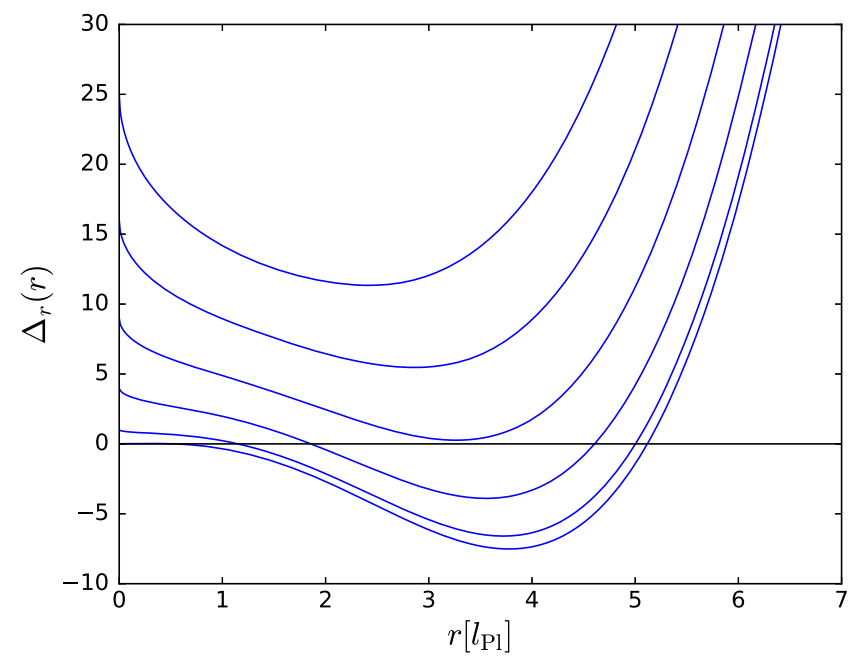

FIG. 4. $\Delta_{r}(r)$ based on the quantum Kretschmann scalar matching for fixed mass $M=5 M_{\mathrm{Pl}}$ and $\Lambda_{0}=-0.1$, but increasing $a=0,1,2,3,4,5$ from bottom to top.

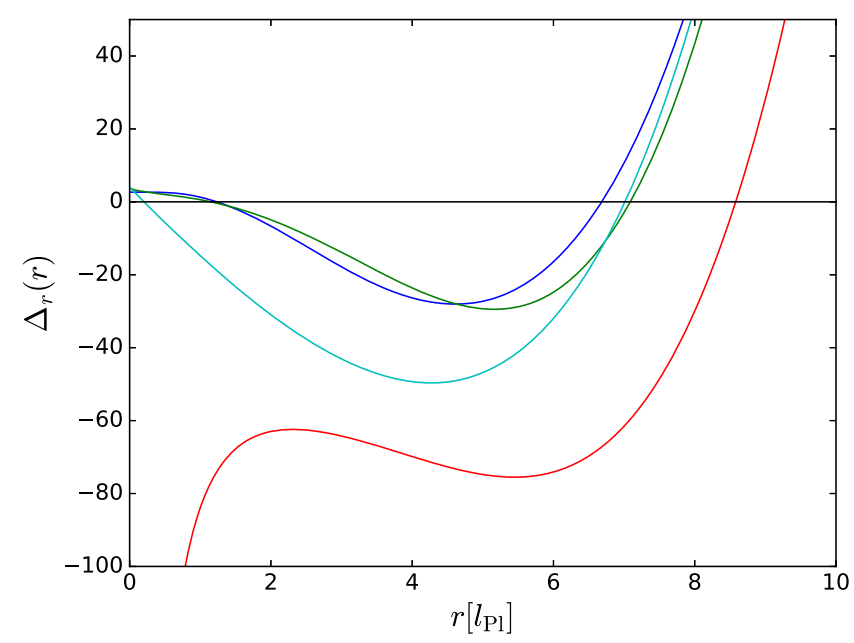

FIG. 5. Comparison of $\Delta_{r}(r)$ for the linear matching in dark blue, the classical Kretschmann setting in red, the quantum Kretschmann setting in green and the classical result from general relativity in light blue, with $M=10 M_{\mathrm{Pl}}, a=2$ and $\Lambda_{0}=-0.1$. Apart from the classical Kretschmann setting, all other matchings agree with the classical position of the outer horizon.

and 9. Varying $m$ controls the position of the two inner horizons via the formation of a minimum, whereas $\Lambda_{0}$ governs the location of the outer horizon. Thereby, the interplay of the amplitudes of $m$ and $\Lambda_{0}$ dictates the number of horizons. Although we cannot provide an analytical condition involving $m$ and $\Lambda_{0}$ for the spacetime exhibiting three horizons, it is suggestive to see it as the generalized version of the condition for a classical Kerr-dS spacetime to have three horizons. This also implies that both quantumimproved spacetimes have two distinct extremal cases: both inner horizons merge at a mass $m=M_{*}$ yielding an extremal black hole inside the cosmological horizon. Or

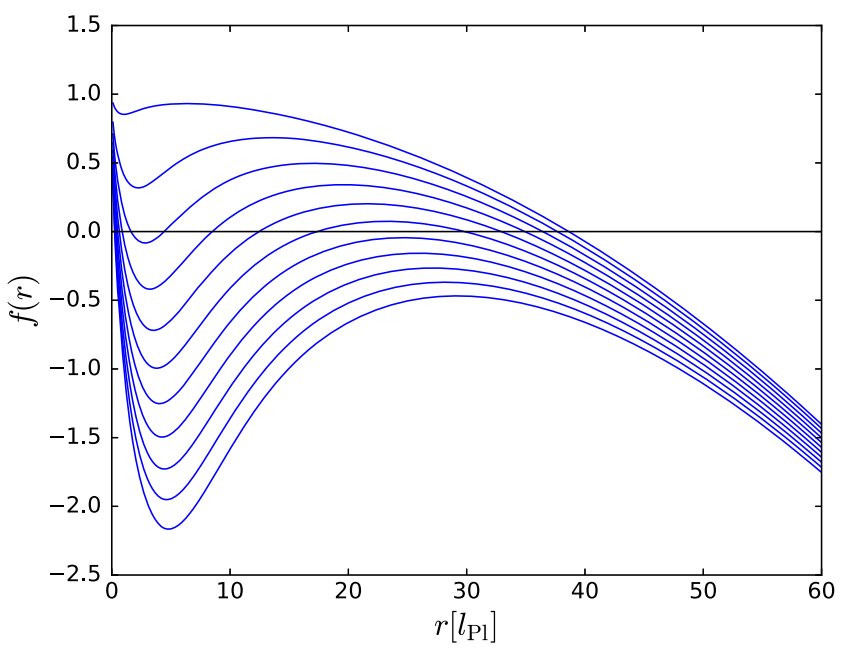

FIG. 6. $f(r)$ for asymptotic dS with $\Lambda_{0}=0.001$ for increasing mass $M=0.1,1,2,3,4,5,6,7,8,9,10 M_{\mathrm{Pl}}$ from top to bottom.

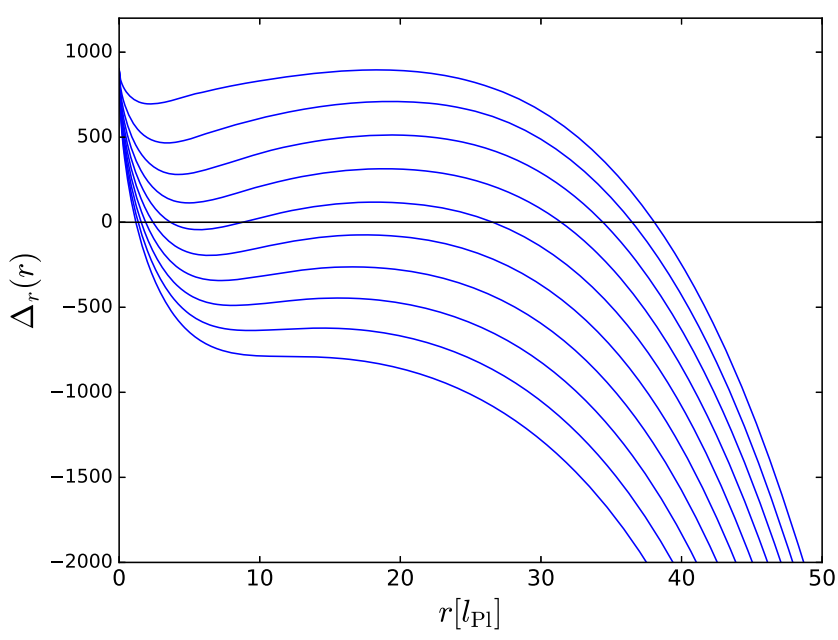

FIG. 7. $\Delta_{r}(r)$ for asymptotic dS with $\Lambda_{0}=0.001$ and $a=30$ for increasing mass $M=1,3,5,7,9,11,13,15,17,19 M_{\mathrm{Pl}}$ from top to bottom.

both outer horizons merge at $m=M^{*}$, forming the largest Schwarzschild/Kerr-dS black hole possible, analogous to the Nariai spacetime.

\section{GLOBAL STRUCTURE, PENROSE DIAGRAMS AND PARTICLE TRAJECTORIES}

In contrast to the classical Schwarzschild-(A)dS and Kerr-(A)dS geometries of general relativity, the quantumimproved counterparts can exhibit a different number of horizons and hence may show a different global structure, depicted in terms of Penrose diagrams. It turns out that both geometries, i.e., one based on the Schwarzschild and the other on the Kerr metric, have the same Penrose diagram. The resulting diagram is equivalent to the classical Reissner-Nordström or Kerr geometry. Hence, the quantum 


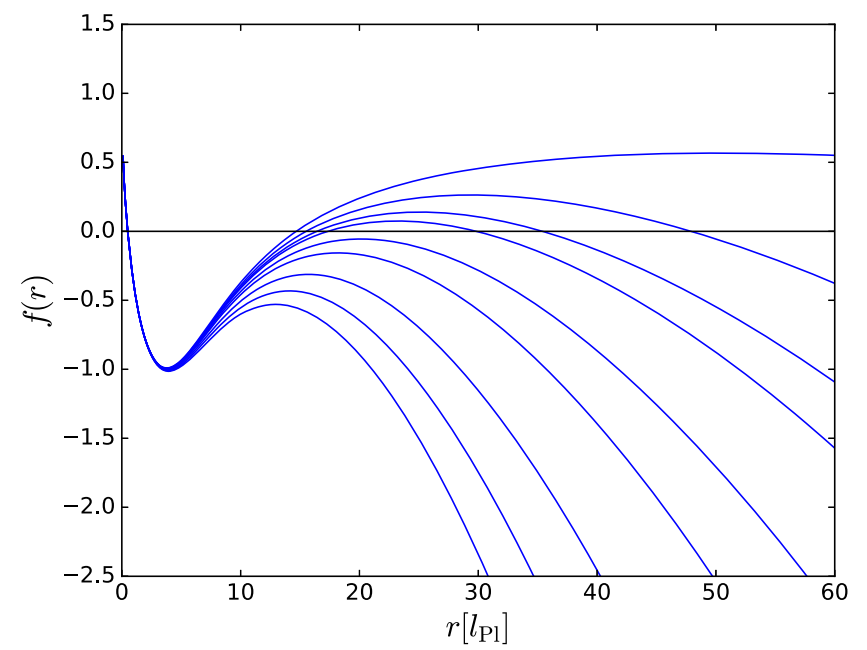

FIG. 8. $f(r)$ for asymptotic dS for increasing $\Lambda_{0}=0.0001$, $0.0005,0.0008,0.001,0.0015,0.002,0.003,0.004,0.005$ from top to bottom and fixed mass $M=5 M_{\mathrm{Pl}}$.

improvements of the metric lead to a unified global structure for quantum-improved black hole spacetimes based on solutions of the Einstein field equations. Yet, as it is shown in Sec. VIC below, particles move differently in each geometry.

We start by determining whether the singularity is timelike, spacelike or null. To that end we compute the norm of the normal vector of a hypersurface of constant $r$ in the limit $r \rightarrow 0$. The norm turns out to be the $r r$-element of the inverse metric $g^{r r}$, yielding

$$
g_{\mathrm{Sch}}^{r r} \stackrel{r \rightarrow 0}{=} 1 \quad \& \quad g_{\mathrm{Kerr}}^{r r} \stackrel{r \rightarrow 0}{\longrightarrow} \frac{1}{\cos ^{2} \theta}
$$

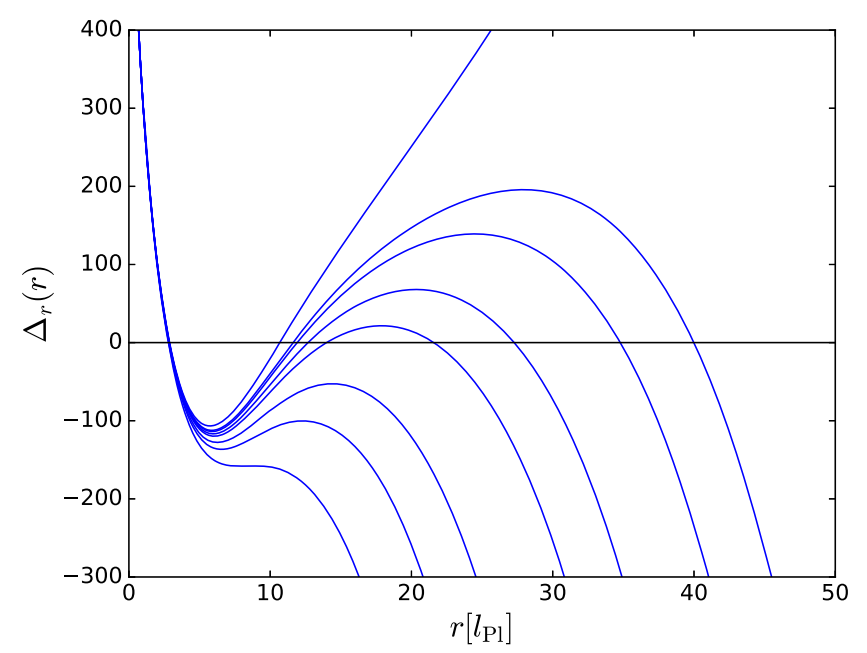

FIG. 9. $\Delta_{r}(r)$ for asymptotic dS for increasing $\Lambda_{0}=0.0001$, $0.0005,0.0006,0.0007,0.0009,0.0015,0.002,0.003$ from top to bottom. Fixed mass $M=5 M_{\mathrm{Pl}}$ and $a=30$.
Hence, the singularity is timelike in both cases, irrespective of whether the spacetime is asymptotically AdS or dS. As is shown in Appendix B, zeros of $f$ and $\Delta_{r}$ correspond to Killing horizons. The succession of sign changes of the lapse function dictates how the hypersurfaces of constant $r$ change from timelike over null to spacelike.

\section{A. Asymptotically anti-de Sitter spacetimes}

The lapse function of Schwarzschild-AdS and the Kerr-AdS spacetime share the same qualitative features, resulting in the same Penrose diagram. The formal construction of the maximally extended spacetime works the same as for the classical Kerr spacetime, for instance see $[22,25]$, but now with an asymptotic AdS-patch. For a mass larger than the critical mass $M_{\mathrm{c}}$, the lapse function has two distinct roots, so the spacetime exhibits two horizons; see Fig. 10. When $m=M_{\mathrm{c}}$, both roots coincide and we find an extremal black hole with just one horizon. For even lower masses, that is $m<M_{\mathrm{c}}$, no horizon is present, but the singularity still exists, cf. Sec. VII, leaving a spacetime with a naked singularity. Later, via a heuristic argument, we will argue that this unphysical spacetime cannot be formed by gravitational collapse.

\section{B. Asymptotically de Sitter spacetimes}

The results for the Schwarzschild- and Kerr-dS geometries agree with each other. The spacetime exhibits two distinguished masses, $M_{*}<M^{*}$, at which two of the possible three horizons merge. Starting with $M_{*}<m<M^{*}$, the spacetime has three distinct horizons, two of them are associated with the black hole and one with the positive cosmological constant on large scales; see Fig. 11. This case is equivalent to the classical Kerr-dS geometry. For $m=M^{*}$, the outer black hole horizon and the cosmological horizon merge. This leaves an extremal spacetime containing a maximally sized black hole, Fig. 12, similar to the Nariai spacetime. For even larger masses, there is just one horizon left; see Fig. 13. On the other end, the de Sitter spacetime contains an extremal black hole if $m=M_{*}$. For $m<M_{*}$, we have a de Sitter geometry containing singularity, which is naked for observers within the cosmological horizon. The construction of the maximally extended spacetime is analogous to the one for the classical Kerr-dS case, described for instance in [22].

\section{Particle trajectories}

In order to investigate whether particles propagate differently in the quantum spacetimes as compared to general relativity, we study their trajectories. Although most new effects in quantum-improved spacetimes happen around the Planck scale, there are possibly deviations from classical trajectories already on length scales well above. Our setup in the following is a test mass with zero angular 


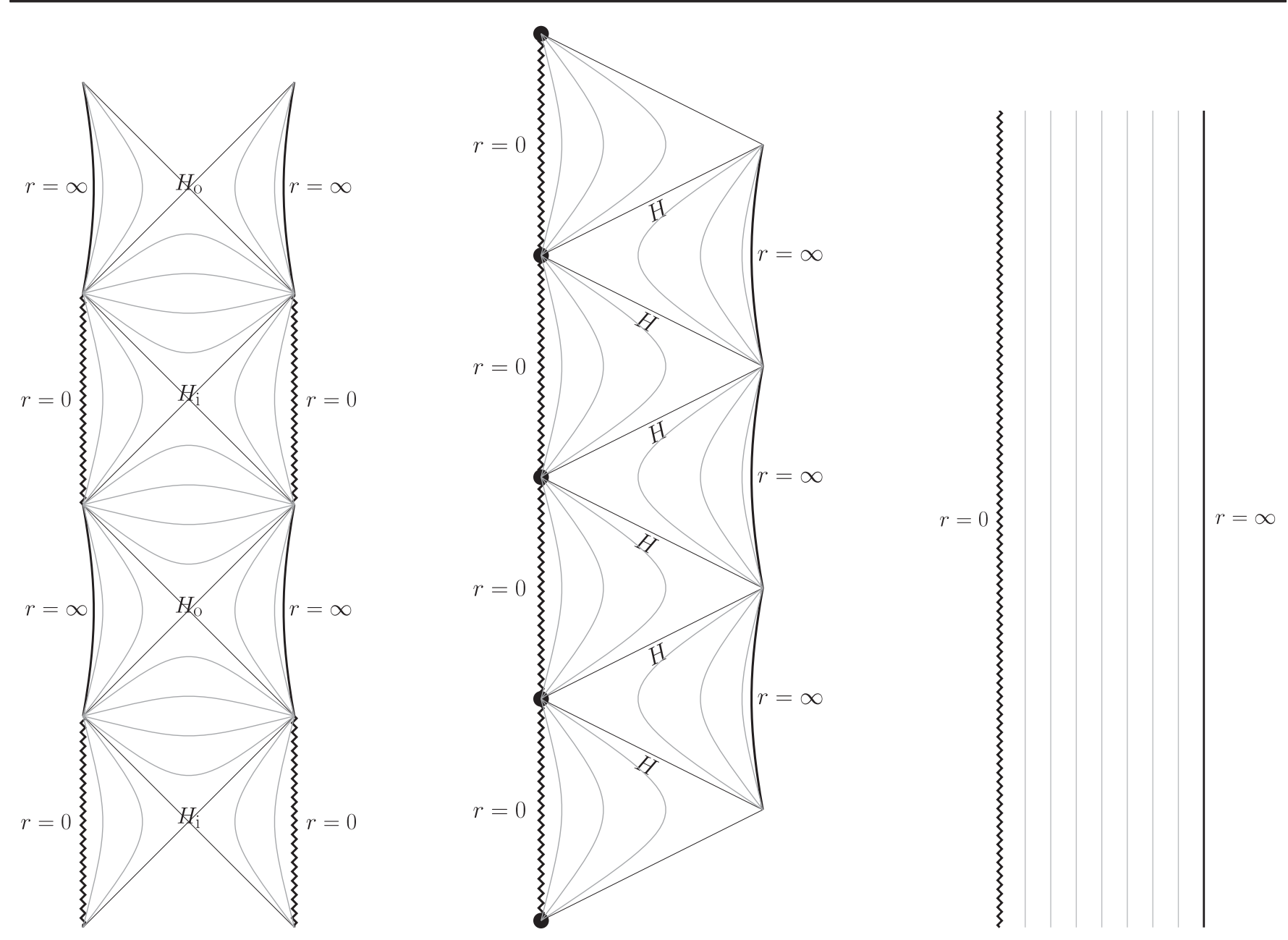

FIG. 10. Penrose diagrams for quantum-improved Schwarzschild-and Kerr-AdS spacetimes. Hypersurfaces $r=$ const are drawn in grey, each diagram can be further extended in vertical direction. To the left the Penrose diagram for the nonextremal black hole with outer horizon $H_{\mathrm{o}}$ and inner horizon $H_{\mathrm{i}}$, the timelike singularity $(r=0)$ and conformal infinity $(r=\infty)$. In the middle the diagram for the extremal geometry with just one horizon $H$. The black dots are not part of the singularity. To the right, the diagram for AdS with a naked singularity at $r=0$.

momentum $L$ along its (timelike) geodesic in a nonextremal geometry, neglecting all backreactions. Furthermore, we are allowed to restrict the motion to the equatorial plane, see [26] for more details. In order to classify orbits into categories, for instance orbits terminating at the central curvature singularity or bound ones, it suffices to study only the change of the radial coordinate.

\section{Schwarzschild}

In the quantum-improved Schwarzschild geometry, the equation for the radial motion of a test mass, starting with zero angular momentum $L$ at some distance $r$ with energy $E$, reads according to (F5)

$$
\dot{r}^{2}=E^{2}-f(r),
$$

where $\dot{r}$ denotes the change of the radial coordinate along the geodesic parametrized by the eigentime. This equation is only dependent on $r$ and can be thought of as an energy equation per unit mass for the total energy $E$ of the test particle in an effective, one-dimensional potential $f(r)$. As was already found in [4] for the asymptotically flat case, possible trajectories are the same as in the classical Reissner-Nordström scenario, thereby differing significantly from a classical Schwarzschild setup. The only difference to the asymptotical flat case arises at large scales, where the effective potential $f(r) \rightarrow \pm \infty$, depending on whether the spacetime is asymptotically de Sitter or anti-de Sitter. Recalling the shape of $f(r)$, e.g., Fig. 1, we note that the effective potential is repulsive close to the singularity. In an asymptotically AdS geometry and for a test mass with energy $E$, the following options are possible, all being bound orbits in radial direction:

(1) If $E$ equals the minimum of the lapse function $f_{\min }$, then the particle is on a circular, stable orbit in the region between the horizons. The radius is 


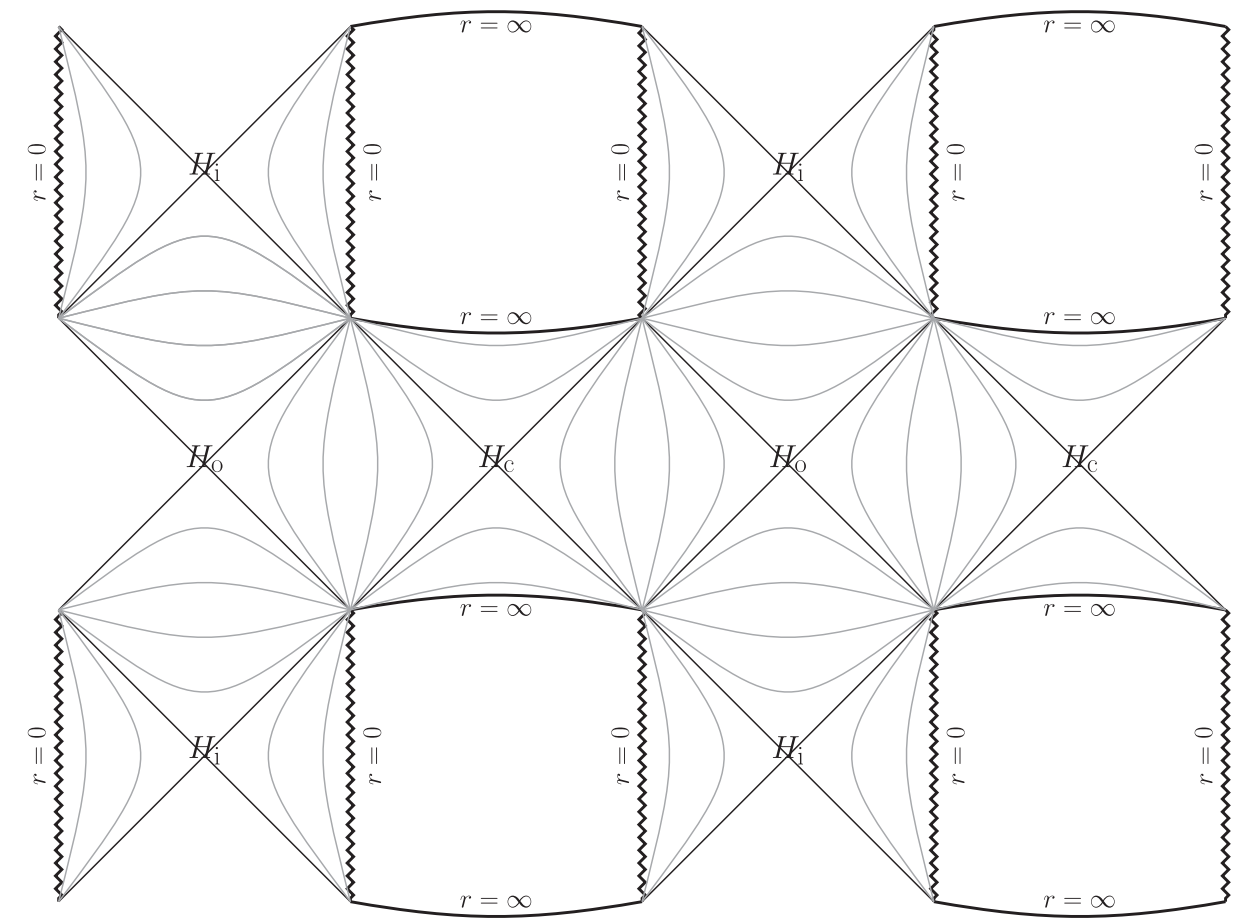

FIG. 11. Penrose diagram for quantum-improved Schwarzschild- and Kerr-dS geometry with the three horizons of a nonextremal black hole configuration. Starting at the timelike singularity at $r=0$, we first cross the inner horizon $H_{\mathrm{i}}$ and then the outer horizon $H_{\mathrm{o}}$ before crossing the cosmological horizon $H_{\mathrm{c}}$ and reaching conformal infinity $r=\infty$. This diagram can be further extended into all directions. Again, $r=$ const hypersurfaces are depicted by grey curves.

determined by the distance where the repulsive singularity balances the repulsive negative asymptotical cosmological constant.

(2) For $f_{\min }<E<0$, the particle is on a bound orbit, remaining in the region between both horizons.

(3) If $0<E<1$, the orbit will again be bound, but now the particle periodically crosses horizons. For instance, first starting in the region outside of the outer horizon, the trajectory will first cross the outer horizon, then the inner one. Because it cannot overcome the repulsive barrier of the singularity, it is bounced back and the radius is increasing again. By crossing another horizon, it will end up in an identical patch of the extended spacetime. This motion continues indefinitely and the particle will travel through infinitely many universes. We will comment on the physicality of this scenario at the end of this section.

(4) If $E>1$, the energy of the particle can overcome the potential barrier and manages to approach the singularity at $r=0$ with nonzero kinetic energy. But in contrast to the classical Schwarzschild-AdS scenario, the particle again follows a path through infinitely many identical universes, reaching the singularity in each of them.

For the case of a nonextremal black hole with asymptotic de Sitter patch, we note that the maximum $f_{\max }$ is always smaller than one. Therefore, we find scenarios one and two from above, but also some differences:

(5) The case $0<E<f_{\max }$ admits a bound orbit, equivalent to scenario three with the outer turning point of the particle being located between the cosmological and the outer black hole horizon, as well as an unbound one beyond the cosmological horizon.

(6) For $E=f_{\max }$, the particle is at rest at the distance, where the attracting force of the black hole balances the attraction generated by the positive cosmological constant on large scales. This is an unstable equilibrium, since small perturbations cause the particle either to move inwards in a similar way to five, or to escape to infinity.

(7) In contrast to all above cases, the orbit is unbound in radial direction for $E>f_{\max }$, and the particle can escape to infinity. Depending on whether or not $E \gtrless 1$, it can reach the singularity at $r=0$.

\section{Kerr}

The equation for the change of the radial coordinate along the geodesic of a test particle with energy $E$ and zero angular momentum $L$ in the equatorial plane of the Kerr geometry reads (cf. (G4)), 


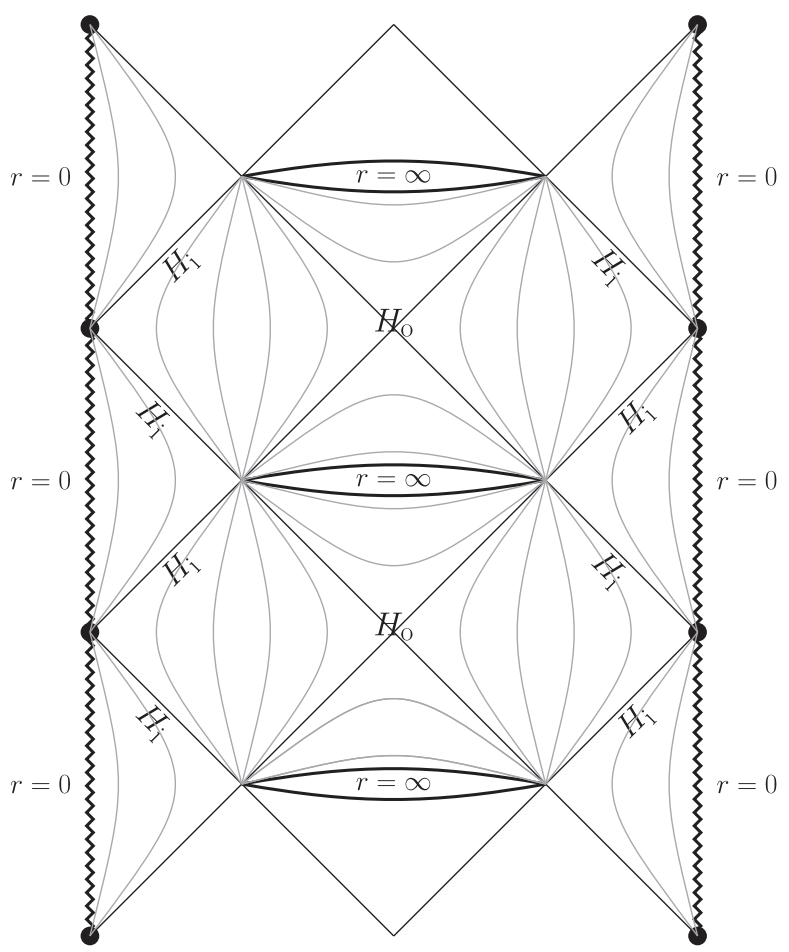

FIG. 12. Penrose diagram for quantum-improved Schwarzschild- and Kerr-dS geometry with the two horizons of an extremal black hole configuration. Starting at the curvature singularity at $r=0$, we first cross the inner horizon $H_{\mathrm{i}}$ and then the outer one $H_{\mathrm{o}}$, before arriving at conformal infinity $r=\infty$. This diagram can be further extended to the top and bottom as well. The black dots are not part of the singularity. The displayed pattern of the $r=$ const hypersurfaces is the one for $m=M^{*}$. For $m=M_{*}$, the hypersurfaces between the horizons become spacelike.

$$
\dot{r}^{2}=R(r):=\frac{E^{2} \Xi^{2}\left[\left(r^{2}+a^{2}\right)^{2}-a^{2} \Delta_{r}\right]-r^{2} \Delta_{r}}{r^{4}},
$$

where we introduced the function $R(r)$ for convenience. For a fixed geometry $\left(G_{0}, \Lambda_{0}, M, a\right)$, the energy $E$ of the

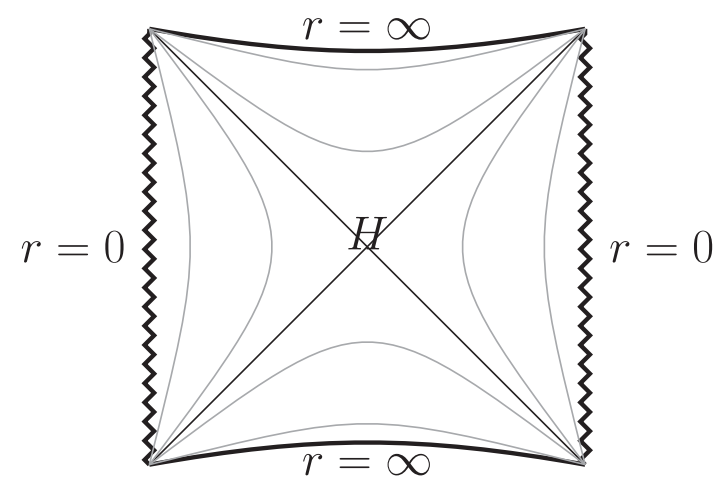

FIG. 13. Penrose diagram for the quantum-improved Schwarzschild-and Kerr-AdS configuration showing only one horizon $H$, always shielding the singularity at $r=0$ from an observer near conformal infinity $r=\infty$. particle determines the allowed orbits. In the following, we continue closely along the more detailed analysis of the classical Kerr-(A)dS geometry carried out in [26]. Since the above equation is quadratic in $\dot{r}$, geodesics always have to satisfy $R(r) \geq 0$. A simple root of $R(r)$ corresponds to a turning point, where the particle comes to rest. A circular orbit of constant $r=r_{0}$ requires both $\dot{r}$ and $\ddot{r}$ to vanish at $r_{0}$, translating via Eq. (14) into the condition of $R(r)$ having an extremum as well as a zero at $r_{0}$. Depending on whether this extremum is a maximum or minimum, the circular orbit will be stable or unstable. Hence, $R(r)$ having at least a double zero at $r_{0}$ is a sufficient condition for a circular orbit.

The function $R(r)$ for Kerr-AdS is displayed in Fig. 14. At large radii, the repulsiveness of the effective AdS spacetime prevents particles from escaping to infinity. There exists a special energy $E_{0}$, above which observers inevitably fall into the singularity along a terminating orbit. For $E=E_{0}$, three types of orbits are possible. $R(r)$ exhibits a double zero at $r_{0}$, allowing for an unstable, circular orbit. For radii larger than $r_{0}$, we find a bound orbit, crossing both horizons. Particles starting at $r<r_{0}$ are accelerated along terminating trajectories and will end up in the singularity. However, if $E<E_{0}$, the double root splits and we find the possibility of having bound orbits as well as terminating ones at radii below the inner horizon. For the smallest energies, $E \rightarrow 0$, the particle moves from horizon to horizon. The only difference for Kerr-dS compared to the AdS case, is that particles can always escape to infinity; see Fig. 15.

The trajectories have been calculated for an idealized, pointlike observer, neglecting any backreaction on the geometry. However, the location of the inner horizon is typically at about the Planck scale, where backreaction effects should be taken into account. The quantumimproved Schwarzschild case turns out to be similar to

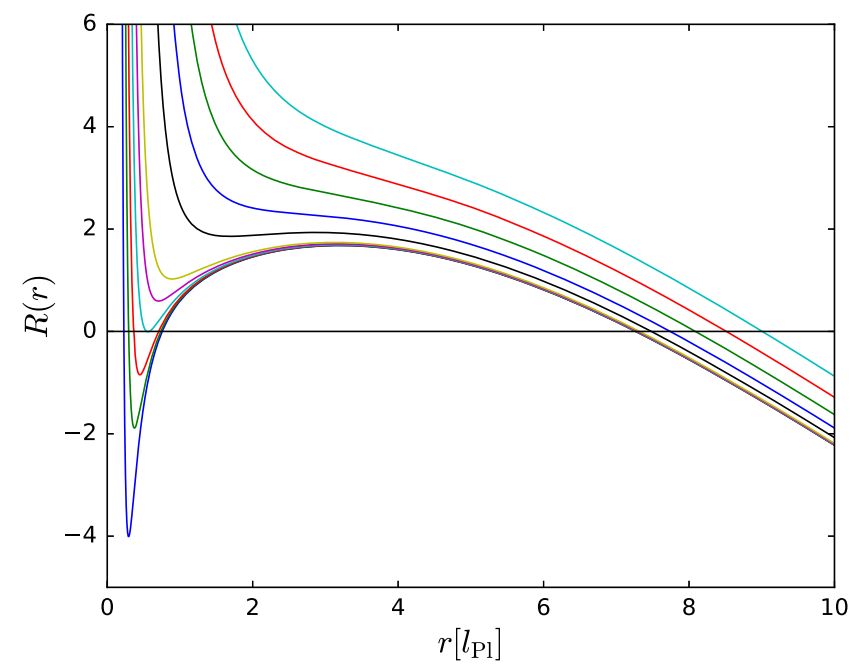

FIG. 14. $R(r)$ from (14) for Kerr-AdS with $G_{0}=1$, $\Lambda_{0}=-0.1, M=10 M_{\mathrm{Pl}}, a=1$ and increasing particle energy $E$ from bottom to top. 


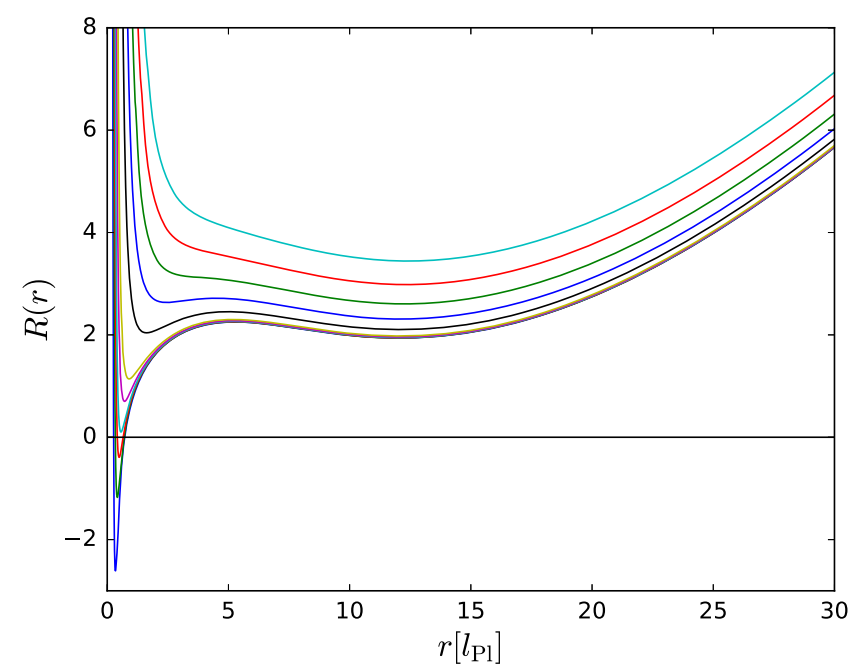

FIG. 15. $R(r)$ from (14) for Kerr-dS with $G_{0}=1, \Lambda_{0}=0.01$, $M=10 M_{\mathrm{Pl}}, a=1$ and increasing particle energy $E$ from bottom to top.

the classical Reissner-Nordström spacetime, for which it was shown that there is a blueshift instability at the inner (Cauchy) horizon. Additionally, it was shown in [27], that perturbations of initial data cause the Cauchy horizon to be replaced by a null singularity. Due to the similarities between the quantum-improved Schwarzschild and the classical Reissner-Nordström spacetime, it is tempting to speculate that the classical findings might also hold for the quantum case. Hence, one has to take the above results with care, especially the many world trajectories. Summarizing, there are differences between the classical and the quantum-improved geometry, but they only become relevant at very small length scales, where the results have to be taken with a grain of salt.

\section{CURVATURE SINGULARITY \& EFFECTIVE ENERGY-MOMENTUM TENSOR}

Since quantum gravity effects become important in high curvature regimes, it is expected that they alter the nature of the curvature singularity at $r=0$. Previous results from asymptotic safe quantum gravity [6-8] and other quantum gravity scenarios, e.g., [28], predict a substantial weakening of the singularity or even its disappearance. A weakening of the singularity manifests itself for instance in changes of the Kretschmann scalar. We compute the Ricci scalar $R$ as well as the Kretschmann scalar $K$ of the quantum-improved geometries in the UV fixed point regime, and compare the findings with the classical result of general relativity. Table I lists the highest degree of divergence of the Ricci and Kretschmann scalar for both investigated geometries for all discussed matchings. Upon comparison with the classical result of general relativity, the consistent quantum scenarios display a weakening of the singularity but not a complete resolution.

In the quantum-improved spacetimes, the Ricci scalar is diverging too, because we have changed the geometry which is not a vacuum solution of the Einstein field equations anymore. In fact, it is a geometry with an effective energy-momentum tensor [29], induced by the running couplings. Using the classical field equations, this effective energy-momentum tensor $T_{\mu \nu}^{\text {eff }}$ can be computed by calculating the Einstein tensor $G_{\mu \nu}$ from the quantumimproved metric,

$$
G_{\mu \nu}+\Lambda_{0} g_{\mu \nu}=: 8 \pi G_{0} T_{\mu \nu}^{\mathrm{eff}}
$$

Note that $T_{\mu \nu}^{\text {eff }}$ is covariantly conserved, assuming a metric connection, $\nabla^{\mu} g_{\mu \nu}=0$, because the Einstein tensor satisfies the Bianchi identity $\nabla^{\mu} G_{\mu \nu}=0$ by construction. However, physical interpretations of this effective energy-momentum tensor in terms of matter have to be drawn with great care. For instance, it turns out that the $T_{r r}^{\mathrm{eff}}$ is diverging at horizons, $f(r)=0$, because $G_{r r}=\frac{f-1+r f^{\prime}}{f r^{2}}$ and $g_{r r}=1 / f(r)$. Additionally, it has been shown in [5], that $T_{\mu \nu}^{\text {eff }}$ in the quantum-improved flat Kerr geometry violates the weak, the null, the strong and the dominant energy condition. We expect similar results in the present case, including the cosmological constant. These observations suggest that quantum gravity contributions to the energy-momentum tensor are of a fundamentally different nature than the ones of conventional matter and should not be interpreted as matter. In fact, the running couplings should be taken into account already on the action level, resulting in different field equations. This is done, for example, in Quantum Einstein Gravity (QEG) [16], based on the quantum-improved Einstein Hilbert action

TABLE I. Ricci scalar $R$ and Kretschmann scalar $K$ for Schwarzschild-and Kerr-AdS for different matchings compared to the classical result.

\begin{tabular}{|c|c|c|c|c|c|c|c|c|}
\hline & Classical & cl. Kretschmann & qu. Kretschmann & Linear & cl. radial path & qu. radial path & cl. geodesic & qu. geodesic \\
\hline$R_{\text {Sch }}$ & $4 \Lambda_{0}$ & $\sim$ const & $\sim r^{-3 / 2}$ & $\sim r^{-2}$ & $\sim$ const & $\sim r^{-2}$ & $\sim$ const & $\sim r^{-3 / 2}$ \\
\hline$K_{\mathrm{Sch}}$ & $\sim r^{-6}$ & $\sim r^{-6}$ & $\sim r^{-3}$ & $\sim r^{-4}$ & $\sim r^{-6}$ & $\sim r^{-4}$ & $\sim r^{-6}$ & $\sim r^{-3}$ \\
\hline$R_{\mathrm{Kerr}}$ & $4 \Lambda_{0}$ & $\sim r^{-3}$ & $\sim r^{-2}$ & $\sim r^{-4}$ & $\sim r^{-4}$ & & $\sim r^{-4}$ & \\
\hline$K_{\text {Kerr }}$ & $\sim r^{-6}$ & $\sim r^{-6}$ & $\sim r^{-4}$ & $\sim r^{-8}$ & $\sim r^{-8}$ & & $\sim r^{-8}$ & \\
\hline
\end{tabular}




$$
S=\int d^{4} x \sqrt{-g}\left[\frac{R-2 \Lambda(r)}{16 \pi G(r)}\right]
$$

The resulting new field equations [30], based on the runnings (3), read the same as (15) with

$8 \pi G_{0} T_{\mu \nu}^{\mathrm{eff}}=-\lambda_{*} k^{2}(r) g_{\mu \nu}+G(r)\left(\nabla_{\mu} \nabla_{\nu}-g_{\mu \nu} \square\right) \frac{1}{G(r)}$.

It has been shown in [31], that the covariant conservation of the effective energy-momentum tensor in QEG is equivalent to the following relation between the running couplings,

$$
R \nabla_{\mu}\left(\frac{1}{G(r)}\right)-2 \nabla_{\mu}\left(\frac{\Lambda(r)}{G(r)}\right)=0 .
$$

This relation is not satisfied by our quantum-improved Schwarzschild-(A)dS and Kerr-(A)dS metrics, meaning that they are not solutions to the new field equations (15) with (18), derived in the Einstein-Hilbert truncation of a potentially more complicated fundamental action.

\section{HORIZON TEMPERATURES AND BLACK HOLE EVAPORATION}

In this section, we first establish the fact, that surface gravities in spacetimes based on the quantum-improved version of the radial path proper distance are divergent, before discussing the Hawking temperatures in the Kretschmann scenario. Finally, we will discuss implications on the black hole evaporation process.

The Hawking temperature of a black hole in flat space received by an observer at infinity is given by $T_{\mathrm{H}}=\frac{\kappa}{2 \pi}$ [32], with surface gravity $\kappa$ of the event horizon. For an observer at finite distance $r$ in the static region outside the black hole, the above expression is modified by a redshift factor,

$$
T_{\mathrm{H}}=\frac{\kappa}{2 \pi} \frac{1}{\sqrt{g(K, K)}},
$$

where $g(K, K)$ is the norm of the static Killing vector $K$. In more general terms, a surface gravity can be assigned to any Killing horizon of a spacetime. Gibbons and Hawking showed in [22], that cosmological horizons also emit radiation which can be detected by an observer in the static spacetime region. In general, emission is a consequence of the observer not being able to access the spacetime hidden behind the horizon(s), thereby being fundamentally unable to measure the quantum state of the complete universe (see [22] for a more detailed discussion). The notion of a horizon temperature only appears to be meaningful for observers in a static spacetime region, since only such observers detect radiation of this temperature. Taking Reissner-Norström as example, this is only the case for the region outside the black hole. In between the horizons, the spacetime is not static anymore and inside the inner horizon, the spacetime is static again, but connected to the singularity. This would require to impose boundary conditions at the singularity, being far from obvious. Hence in the following, we only refer to a horizon having a temperature, if the horizon is the boundary of a static region, not connected to the singularity. In Appendix B, horizons in the quantum-improved spacetime are shown to be Killing horizons, thus a surface gravity can be assigned to each of them.

Technically, the surface gravity $\kappa$ of a Killing horizon can be computed by taking the covariant derivative of the norm of the Killing vector, or alternatively via a periodicity in Euclidean time introduced in [33]. In any case, we find

$$
\kappa_{\mathrm{Sch}}=\frac{1}{2}\left|f^{\prime}\left(r_{0}\right)\right| \quad \& \quad \kappa_{\mathrm{Kerr}}=\frac{1}{2} \frac{\left|\Delta_{r}^{\prime}\left(r_{0}\right)\right|}{\left(r_{0}^{2}+a^{2}\right)},
$$

$r_{0}$ being the radial coordinate of the horizon. Since horizons are zeros of $f(r)$ and $\Delta_{r}(r)$, respectively, $(\mathrm{C} 4)$ implies that the derivative of the proper distance $D^{\prime}(r)$ diverges at the horizons for the quantum version of the radial path. As addressed in Appendix D in detail, this does not necessarily mean that the proper distance itself is diverging at a horizon, unless the horizon is extremal. But computing the surface gravity explicitly via (20) generates the following terms, proportional to $D^{\prime}(r)$, and therefore diverging at the horizons,

$$
\begin{aligned}
& f^{\prime}(r) \sim \frac{2}{3 r^{2}}\left(-\frac{6 G_{0}^{2} g_{*} \lambda_{*} M r D(r)}{\left(g_{*} \lambda_{*} D^{2}(r)+G_{0}\right)^{2}}+\frac{r^{4}}{D^{3}(r)}\right) D^{\prime}(r), \\
& \Delta^{\prime}(r) \sim\left(\frac{2 r^{2}\left(a^{2}+r^{2}\right)}{3 D^{3}(r)}-\frac{4 G_{0}^{2} g_{*} \lambda_{*} M r D(r)}{\left(g_{*} \lambda_{*} D^{2}(r)+G_{0}\right)^{2}}\right) D^{\prime}(r) .
\end{aligned}
$$

The terms in the brackets are in general nonvanishing at the horizons. In particular, this holds also for arbitrary large masses in the classical regime, where it is known that the surface gravity and Hawking temperature stays finite. This is the main reason why we consider the scale identification based on the quantum radial path as unphysical. In contrast, along with the proper distance based on a geodesic, the construction based on the Kretschmann scalar shows no divergent behavior at the horizons and therefore leads to finite Hawking temperatures.

Next, we discuss the mass dependence of the surface gravities, focusing on the quantum Kretschmann scenario from now on. It suffices to look at the slope of the lapse function at each horizon, since it is proportional to the surface gravity. The results for Schwarzschild-AdS and Schwarzschild-dS can be found in Figs. 16 and 17, the plots for the Kerr cases are qualitatively the same. The whole evolution, appearance and disappearance of horizons is driven by the formation of a minimum of the lapse function. The quantum-improved Schwarzschild-AdS spacetime exhibits no horizon up to the critical mass $M_{\mathrm{c}} \approx 1.2 M_{\mathrm{Pl}}$. At $M=M_{\text {crit }}$, the minimum of the lapse function is at zero, 


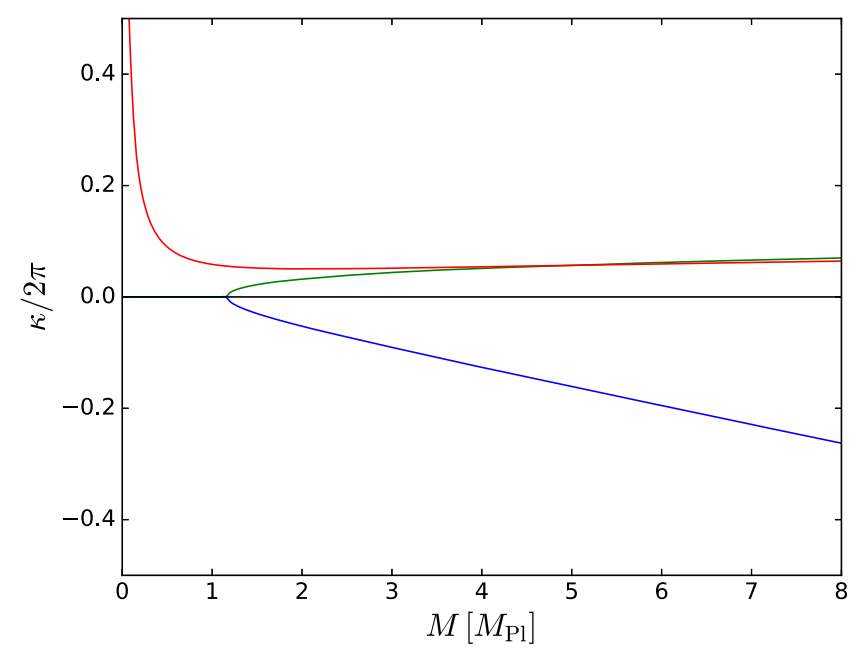

FIG. 16. $f^{\prime}(r)$ as function of the mass $M$ for the quantum improved Schwarzschild-AdS geometry for $\Lambda_{0}=-0.1$. Inner horizon in blue, outer horizon in green. The outer horizon agrees with the temperature of the event horizon in general relativity in red for large masses. Taking absolute values yields the surface gravities.

hence the slope is zero and so are the surface gravities. For growing mass, the slope becomes steeper because the minimum expands, thus the surface gravities grow in amplitude. In contrast, $\kappa_{\mathrm{cl}}$ in general relativity diverges for $M \rightarrow 0$. However, the surface gravity of the outer horizon matches the classical one for sufficiently large masses. The Schwarzschild-dS scenario can have up to three horizons and two special masses, $M_{*} \approx 2 M_{\mathrm{Pl}}$ and $M^{*} \approx 5.8 M_{\mathrm{Pl}}$, at which two of the three horizons merge. Starting in the $M<$ $M_{*}$ regime, there is no black hole, but only the cosmological

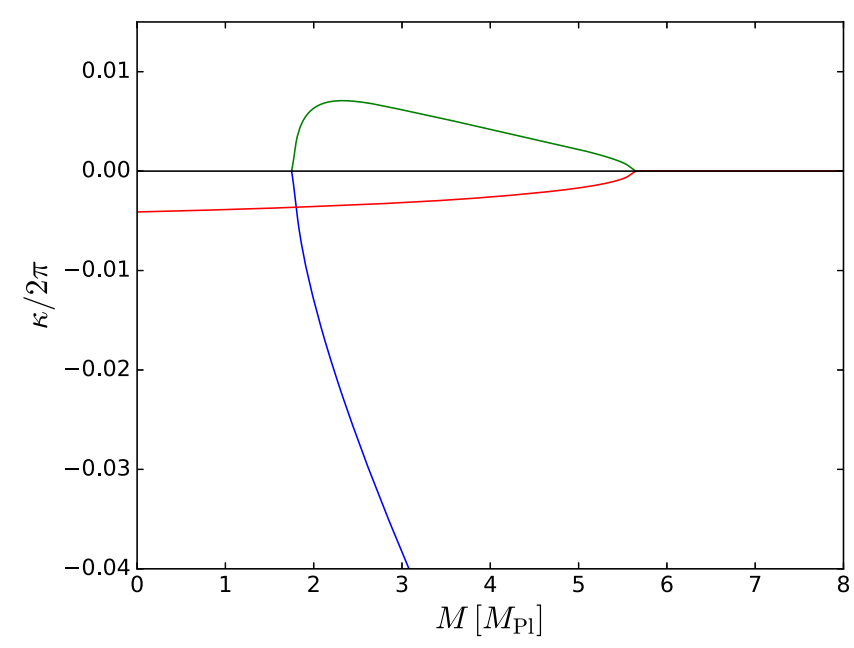

FIG. 17. $f^{\prime}(r)$ as function of the mass $M$ for the quantum improved Schwarzschild-dS geometry for $\Lambda_{0}=0.001$. The cosmological horizon in red, the inner black hole horizon in blue and the outer black hole horizon in green. Taking absolute values yields the surface gravities. horizon. The case $M=M_{*}$ corresponds to the case $M=M_{\mathrm{c}}$ from above. For $M_{*}<M<M^{*}$, there are three horizons and the back hole gets bigger for increasing mass, until $M=M^{*}$, when the black hole has reached its maximal size and its outer horizons merges with the cosmological horizon to an extremal horizon with zero temperature.

In AdS spacetimes, an observer in the static region could only measure a temperature coming from the black holes' event horizon, whereas in dS spacetimes, the observer would measure a mixture of two thermal spectra at different temperatures, one coming from the back hole and one from the cosmological horizon. In the static region outside the black hole, one valid choice for the Killing vector in (19) is $K=\partial / \partial t$, yielding $g(K, K)=g_{t t}$. In the Schwarzschild geometries, this implies that an observer located at a horizon would measure an infinite temperature, in accordance with general relativity. In Schwarzschild-AdS, the temperature drops to zero for an infinitely distant observer, as $g_{t t}$ diverges.

In the dS-scenario, there exists a distance between the horizons, at which the observed temperature becomes minimal, s because $f$ has a maximum. In the Kerr geometries, $\partial / \partial t$ is timelike only outside the ergoregion. A static Killing vector field for the entire region outside the black hole can be obtained by linearly combining the two Killing vectors of a Kerr spacetime; see Appendix B. Since all above observations equally apply for classical as well as quantum improved spacetimes, the is no qualitative difference for an observer measuring horizon temperatures in a classical or a quantum spacetime, except in the Planckian regime.

As final point, we would like to address the black hole evaporation process. A standard mechanism to form black holes is gravitational collapse. If the mass of a collapsing object is larger than the Tolman-Oppenheimer-Volkoff mass around $2 M_{\odot}$, no other force can counterbalance gravity and the object collapses to form a black hole. Assuming that a macroscopic Schwarzschild or Kerr black hole has formed via this process, well above the critical mass, it will emit Hawking radiation and thereby lose energy. This causes the black hole to shrink steadily, as its mass is decreasing. This process continues, until the critical mass $M_{\text {crit }}$ is reached. The temperature then becomes zero and therefore the radiation stops. Hence, the naked singularity case with $M<M_{\text {crit }}$ can never be reached via this process and we end up with a zero temperature, Plancksized, extremal black hole, often referred to as remnant. This remnant serves as shield, guaranteeing that the cosmic censorship conjecture remains satisfied. However, in [34] it was shown that extremal black hole configurations with zero temperature suffer from an instability at the extremal horizon. Remnant end points were also found in other studies within asymptotic safety [7,10] and beyond [35]. Based on a classical expression for the proper distance it has been shown in $[6,8]$ that the Schwarzschild-AdS black hole evaporates completely. A more suitable setup to 
discuss the evaporation process is given by the dynamical Vaidya spacetime, used in [10]. There, a Planck-sized, cold remnant as an end point has been found.

\section{SUMMARY}

In this work, the quantum-improved Kerr-(A)dS black hole was studied for the first time within a self-consistent scale identification procedure. The latter is based on the Kretschmann scalar. The Kerr-(A)dS geometry also includes the Schwarzschild-(A)dS, as well as ordinary Schwarzschild and Kerr spacetimes as special cases, by setting either the rotation parameter $a$ or the cosmological constant $\Lambda_{0}$ to zero.

Both quantum-improved geometries show the same global structure in terms of a timelike curvature singularity at $r=0$ and the same number of horizons. Furthermore, it has also been shown that the outer black hole horizon corresponds to the classical black hole event horizon. The timelike character of the singularity at $r=0$ in principle allows particles to avoid the singularity. The quantum corrections to the classical metric render the singularity less divergent, but none of the studied scenarios was able to resolve it completely. However, this singularity will always be dressed by a horizon, such that there is no violation of the cosmic censorship conjecture.

The horizons being Killing horizons admit a temperature, causing the black hole to evaporate. In the Planckian regime, however, the heat capacity of a tiny black hole stays positive, $\frac{\partial T}{\partial M}>0$, in contrast to the classical case. Thus, the evaporation process comes to an end when the Hawking temperature of the black hole is zero, leaving an extremal, cold, Planck-sized remnant, serving as cosmic censor. This is a thermodynamically stable end point because any additional mass absorbed by the black hole will radiate away until the temperature is again zero. It would be interesting to see what implications for the black hole information paradox can be drawn from the generic existence of such remnants.

\section{ACKNOWLEDGMENTS}

We thank Alfio Bonanno, Kevin Falls, Domenico Giulini, and Alessia Platania for discussions. This work is supported by ExtreMe Matter Institute (EMMI) and is part of and supported by the DFG Collaborative Research
Centre "SFB 1225 (ISOQUANT)" and also by the DFG Research Training Group "Models of Gravity."

\section{APPENDIX A: CHOICE OF SCALE IDENTIFICATION}

Here we motivate our choice for $k(r)$ in (5). Inserting the general parametrization $k(r)=\xi / D(r)$, into (C5), we are left with

$$
\begin{aligned}
& f(r)=1-\frac{2 M}{r} \frac{g(r) D^{2}(r)}{\xi^{2}}-\frac{r^{2}}{3} \frac{\lambda(r) \xi^{2}}{D^{2}(r)} \\
& \underset{r \rightarrow 0}{\stackrel{\mathrm{UV}}{\approx}} 1-\frac{2 M}{r} \frac{g_{*} D^{2}(r)}{\xi^{2}}-\frac{r^{2}}{3} \frac{\lambda_{*} \xi^{2}}{D^{2}(r)}, \\
& \Delta_{r} \underset{r \rightarrow 0}{\stackrel{\mathrm{UV}}{\approx}}\left(r^{2}+a^{2}\right)\left(1-\frac{r^{2}}{3} \frac{\lambda_{*} \xi^{2}}{D^{2}(r)}\right)-\frac{2 M}{r} \frac{g_{*} D^{2}(r)}{\xi^{2}} .
\end{aligned}
$$

The numerical values of $g_{*}$ and $\lambda_{*}$ depend on the particular RG-trajectory and parametrization we have chosen and therefore cannot be physical observables. However, the product $g_{*} \lambda_{*}$ is an observable and hence independent of the particular choice of the RG-trajectory. Its magnitude turns out to be $g_{*} \lambda_{*} \approx 0.1$, e.g., in $[16,20]$. In this light, we have two choices for $\xi$ in order to make (A1) solely dependent on $g_{*} \lambda_{*}$,

$$
\xi^{2}=g_{*} \quad \text { or } \quad \xi^{2}=\frac{1}{\lambda_{*}} .
$$

Thus, in (5) we have chosen the second of the two equivalent options. Varying $\xi$ for a fixed geometry $\left(G_{0}\right.$, $\Lambda_{0}, m, a$ ), which is effectively done also in the quantum Kretschmann scenario by introducing $\chi$, turns out to have only a weak impact on the position of the inner horizon. Since it is typically located at small radii, we recall from Table II, that varying $\xi$ mildly modifies the UV-limit. Furthermore, we have an upper limit $\chi<(3 / 8)^{1 / 4}$.

\section{APPENDIX B: KILLING HORIZONS}

In this section, we review the formal proof that every zero of $\Delta_{r}(r)$ in (7) is a Killing horizon. This implies that a constant surface gravity and thereby a temperature can be

\begin{tabular}{|c|c|c|c|c|c|c|}
\hline & \multicolumn{2}{|c|}{ Kretschmann } & \multicolumn{2}{|c|}{ Radial path } & \multicolumn{2}{|c|}{ Geodesic path } \\
\hline & Classic & Quantum & Classic & Quantum & Classic & Quantum \\
\hline Schwarzschild & $\frac{1}{r^{3 / 2}}$ & $\left(\chi^{-4}-8 / 3\right)^{1 / 8} r^{3 / 4}$ & $\frac{2}{3 \sqrt{2 G_{0} M}} r^{3 / 2}$ & $\frac{2}{\sqrt{3}} r$ & $\frac{\pi}{2 \sqrt{2 G_{0} M}} r^{3 / 2}$ & $\left(\frac{67}{18 M g_{*} \lambda_{*}}\right)^{1 / 4} r^{3 / 4}$ \\
\hline Kerr & $\overline{3^{1 / 4} 2 \chi \sqrt{M G_{0}}} r$ & $\left(\frac{\lambda}{48 M^{2}\left(g_{*} \lambda_{*}\right)^{2}}\right) \quad r^{3 / 4}$ & $\frac{r^{2}}{2 a}$ & 0 & $\frac{\pi}{4 a} r^{2}$ & 0 \\
\hline
\end{tabular}
associated to each horizon. The Schwarzschild-(A)dS case is automatically contained by taking $a \rightarrow 0$.

TABLE II. UV-limits $(r \rightarrow 0)$ of $D(r)$ for all investigated matchings. 

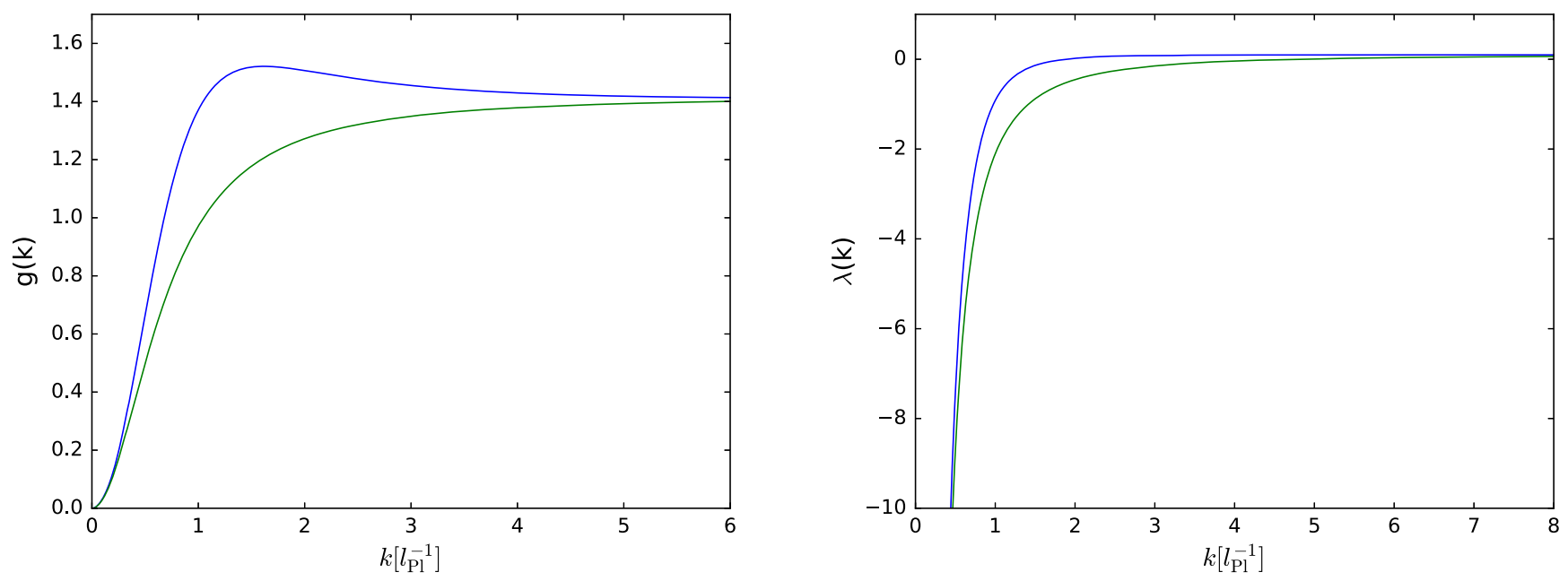

FIG. 18. Running of the dimensionless couplings $g$ and $\lambda$ as a function of momentum scale $k$ for the analytical expressions from (3) in green and from a fourth-order vertex expansion based on [20] in blue. Both approach their UV fixed point values, $g_{*}=1.4 \& \lambda_{*}=0.1$, for $k \rightarrow \infty$.

Starting from the Kerr-(A)dS metric (7), assume that $\Delta_{r}(r)$ has $j$ positive roots, i.e., can be written as

$$
\Delta_{r}(r)=\prod_{i=0}^{j}\left(r-r_{i}\right) \text { with } 0 \leq r_{0} \leq r_{1}<\ldots \leq r_{j} .
$$

The horizons are the hypersurfaces $r=r_{i}=$ const. Since the spacetime is axisymmetric and stationary, we have two commuting Killing vector fields: $\left(\frac{\partial}{\partial t}\right)^{a}$ is stationary, at least in some region of the spacetime, and $\left(\frac{\partial}{\partial \phi}\right)^{a}$ manifests the symmetry axis. We now have to construct a Killing vector field $\xi^{a}$, that is normal to, and null on these horizon hypersurfaces. The most general form for $\xi^{a}$ would be a linear combination of both Killing vector fields,

$$
\xi^{a}=\left(\frac{\partial}{\partial t}\right)^{a}+\alpha\left(\frac{\partial}{\partial \phi}\right)^{a}
$$

with a constant $\alpha$. We will fix this constant later by requiring that $\xi^{a}$ should vanish at the horizons. But first, we must change from Boyer-Lindquist coordinates (7), to coordinates that leave the metric regular at the horizons. Such coordinates are induced by the principal null directions of the spacetime. The Kerr-(A)dS spacetime is of algebraic type $\mathrm{D}$, thus admits two distinct principal null directions, referred to as ingoing and outgoing. They can be represented in Boyer-Lindquist coordinates by the following vectors,

$$
n_{ \pm}^{\mu}=\left(\frac{r^{2}+a^{2}}{\Delta_{r}} \Xi, \pm 1,0, \frac{a}{\Delta_{r}} \Xi\right),
$$

where +1 is outgoing and -1 ingoing. They now induce outgoing and ingoing coordinates, being the Kerr-(A)dS counterparts of Kerr-coordinates in flat space. We will select the outgoing version, but in principle we could also work with ingoing ones. The outgoing Kerr-(A)dS coordinates $(v, \chi)$ are defined as,

$$
\begin{aligned}
& \mathrm{d} v=\mathrm{d} t+\Xi \frac{r^{2}+a^{2}}{\Delta_{r}} \mathrm{~d} r \\
& \mathrm{~d} \chi=\mathrm{d} \phi+\Xi \frac{a}{\Delta_{r}} \mathrm{~d} r .
\end{aligned}
$$

Inserting these back into (7), leaves us with the metric in terms of Kerr-(A)dS coordinates $(v, r, \theta, \chi)$,

$$
\begin{aligned}
\mathrm{d} s^{2}= & -\frac{1}{\rho^{2} \Xi^{2}}\left(\Delta_{r}-\Delta_{\theta} a^{2} \sin ^{2} \theta\right) \mathrm{d} v^{2}+\frac{2}{\Xi} \mathrm{d} v \mathrm{~d} r \\
& -\frac{2 a \sin ^{2} \theta}{\rho^{2} \Xi^{2}}\left(\left(r^{2}+a^{2}\right) \Delta_{\theta}-\Delta_{r}\right) \mathrm{d} v \mathrm{~d} \chi-\frac{2 a \sin ^{2} \theta}{\Xi} \mathrm{d} \chi \mathrm{d} r \\
& +\frac{\sin ^{2} \theta}{\rho^{2} \Xi^{2}}\left(\Delta_{\theta}\left(r^{2}+a^{2}\right)^{2}-\Delta_{r} a^{2} \sin ^{2} \theta\right) \mathrm{d} \chi^{2}+\frac{\rho^{2}}{\Delta_{\theta}} \mathrm{d} \theta^{2} .
\end{aligned}
$$

One can check that (B5) reduces to Kerr coordinates for $\Lambda=0$. The Killing vector field $\xi^{a}$ now reads

$$
\xi^{a}=\left(\frac{\partial}{\partial v}\right)^{a}+\alpha\left(\frac{\partial}{\partial \chi}\right)^{a}
$$

Requiring that $\xi^{a}$ is null on the horizons $r=r_{i}$ yields

$$
\begin{aligned}
\left.\xi^{2}\right|_{r=r_{i}} & =\left[g_{v v}+2 \alpha g_{v \chi}+\alpha^{2} g_{\chi \chi}\right]_{r=r_{i}} \\
& =\frac{\Delta_{\theta} \sin ^{2} \theta}{\rho_{i}^{2} \Xi^{2}}\left[a-\alpha\left(r_{i}^{2}+a^{2}\right)\right]^{2} \stackrel{!}{=} 0
\end{aligned}
$$

and therefore 


$$
\alpha=\frac{a}{r_{i}^{2}+a^{2}}
$$

Thus, we have found a family of vector fields $\left(\xi^{a}\right)_{i}$, being null at one horizon at a time. In order to show that the hypersurfaces $r=r_{i}$ are Killing horizons, it remains to be checked if $\xi^{a}$ is hypersurface orthogonal, i.e., $\xi_{a}=\xi_{\mu} \mathrm{d} x^{\mu} \sim$ $\mathrm{d} r$ evaluated at the horizon,

$\left.(\xi)_{a}\right|_{r=r_{i}}=\left[g_{\mu \nu} \xi^{\nu} \mathrm{d} x^{\mu}\right]_{r=r_{i}}=\frac{1}{\Xi}\left(1-\frac{a^{2} \sin ^{2} \theta}{r_{i}^{2}+a^{2}}\right) \mathrm{d} r$,

with all other components vanishing. In summary, we are able to construct a Killing vector field $\xi^{a}$ which is null on, and normal to each horizon hypersurface $r=r_{i}$, and hence have shown that the horizons corresponding to the roots of $\Delta_{r}$ are indeed Killing horizons.

\section{APPENDIX C: OTHER MATCHINGS}

\section{Linear matching}

The simplest scaling is based on a dimensional analysis,

$$
D_{\text {Lin }}(r)=r
$$

which has already been adopted for instance in [4]. In the case of an identically vanishing cosmological coupling, is the IR-limit of the classical proper distance along a radial path [7]. But this matching does not take physical scales of the underlying spacetime into account, for instance the black hole scales given by $M \& a$, or scales induced by the gravitational or the cosmological coupling. Nevertheless, this function already gives rise to many phenomena observed for more complicated choices and hence can serve as a toy model. The results for the lapse functions based on the linear matching can be found in Figs. 19 and 20.

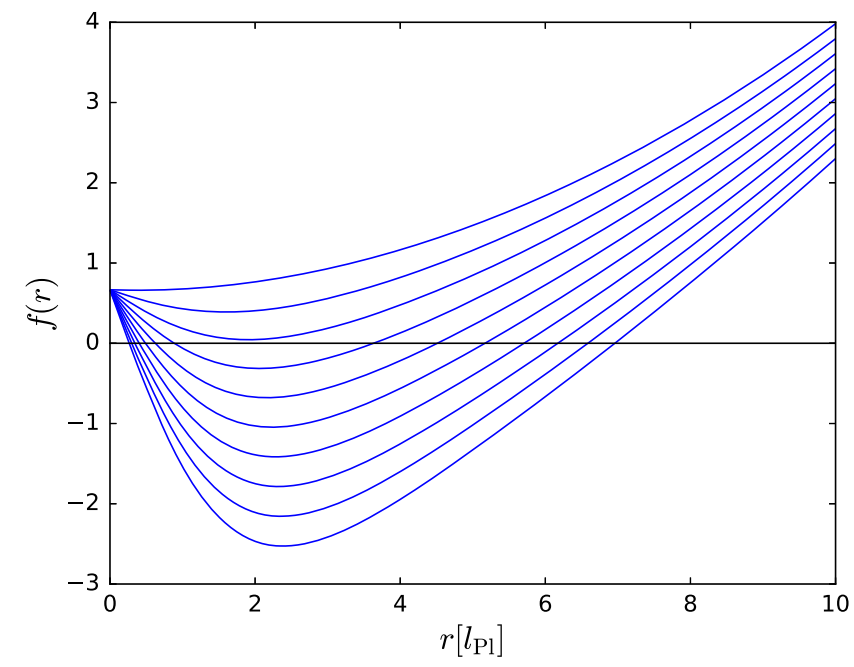

FIG. 19. $f(r)$ from (6) based on the linear matching for increasing mass from top to bottom, with $\Lambda_{0}=-0.1$, $M=0.1,1,2,3,4,5,6,7,8,9,10 M_{\mathrm{Pl}}$.

\section{Proper Distances}

We can also use the proper distance along a curve $\mathcal{C}$ in spacetime to specify $D(r)$,

$$
D(r)=D_{\text {prop }}=\int_{\mathcal{C}} \sqrt{\left|g_{\mu \nu} \mathrm{d} x^{\mu} \mathrm{d} x^{\nu}\right|}
$$

This definition is diffeomorphism invariant and encodes the spacetime structure, since the gravitational and cosmological coupling typically appear in the metric. In most cases in the literature, e.g., [6-8], the gravitational as well as cosmological coupling have been fixed to be constants, for instance the IR-values $\Lambda_{0}$ and $G_{0}$. However, since the FRG-flow generically gives rise to running couplings, it is more natural and consequent to consider this running also in the above integral, thus $G \rightarrow G(r)$ and $\Lambda \rightarrow \Lambda(r)$. In the following, this quantum improvement procedure of proper distances is extended to Schwarzschild- and Kerr-(A)dS geometries. We will provide expressions for the proper distance along a radial path and along the geodesic of a radially infalling observer, both for constant, as well as running $G$ and $\Lambda$. Additionally, the UV-limit of each proper distance is obtained, cf. Table II.

\section{a. Radial path}

Inspired by the symmetry of the spacetime, we first take the following radial path from 0 to $r$ as integration contour $\mathcal{C}$ in (C2),

$$
\begin{aligned}
& \mathcal{C}_{\text {Schw-(A)dS }}: \mathrm{d} t=\mathrm{d} \Omega=0 \\
& \mathcal{C}_{\text {Kerr-(A)ds }}: \mathrm{d} t=\mathrm{d} \phi=\mathrm{d} \theta=0 \text { and } \theta=\pi / 2 .
\end{aligned}
$$

The restriction to the equatorial plane in the Kerr case is done for the sake of simplicity. Driven by the results of [5]

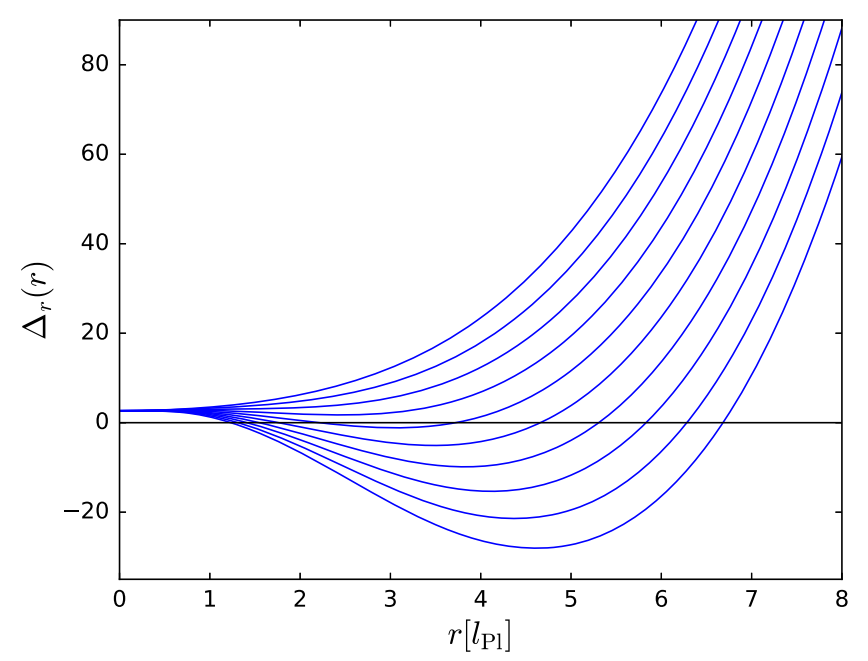

FIG. 20. $\Delta_{r}(r)$ from (8) based on the linear matching for increasing mass from top to bottom, with $\Lambda_{0}=-0.1, a=2$ and $M=0.1,1,2,3,4,5,6,7,8,9,10 M_{\mathrm{Pl}}$. 
for the flat Kerr geometry, we assume that the varying $\theta$ will not alter our results qualitatively. Applying the above integration paths to $(\mathrm{C} 2)$ yields,

$$
\begin{gathered}
D_{\mathrm{Sch}}(r)=\int_{0}^{r} \mathrm{~d} \tilde{r} \sqrt{\left|g_{\tilde{r} \tilde{r}}\right|}=\int_{0}^{r} \mathrm{~d} \tilde{r} \frac{1}{\sqrt{|f(\tilde{r})|}}, \\
D_{\mathrm{Kerr}}(r)=\int_{0}^{r} \mathrm{~d} \tilde{r} \sqrt{\left|g_{\tilde{r} \tilde{r}}\right|}=\int_{0}^{r} \mathrm{~d} \tilde{r} \sqrt{\frac{\tilde{r}^{2}}{\left|\Delta_{r}(\tilde{r})\right|}},
\end{gathered}
$$

with the lapse functions

$$
\begin{aligned}
f(r) & =1-\frac{2 G M}{r}-\frac{\Lambda}{3} r^{2} \quad \text { and } \\
\Delta_{r}(r) & =\left(r^{2}+a^{2}\right)\left(1-\frac{\Lambda}{3} r^{2}\right)-2 M G r
\end{aligned}
$$

In the following, this scenario with constant $G$ and $\Lambda$ will be referred to as the classical radial path because the spacetime underlying the integral is a classical black hole geometry with a cosmological constant.

Alternatively, we account for the running of the couplings already in the proper distance, referred to as the quantum radial path, with $G=G(r)$ and $\Lambda=\Lambda(r)$ in the above integrals. This turns (C4) into integral equations for $D(r)$, which can be transformed into a differential equation by taking a derivative with respect to $r$. One can then easily see that the derivative of $D(r)$ diverges at every horizon, where $f(r)$ and $\Delta(r)$ vanish. Using the fixed point behavior of $G$ and $\Lambda$ in the UV, these differential equations read for small $r$,

$$
\begin{aligned}
& D_{\text {sch,qu }}^{\prime}(r)=\frac{1}{\sqrt{\left|1-2 M g_{*} \lambda_{*} \frac{D_{\text {sch,qu }}^{2}(r)}{r}-\frac{r^{2}}{3 D_{\text {sch,qu }}^{2}(r)}\right|}}, \\
& D_{\text {kerr,qu }}^{\prime}(r)=\frac{1}{\sqrt{\left|1+\frac{a^{2}}{r^{2}}-\frac{r^{2}}{3 D_{\text {kerr,qu }}^{2}(r)}-\frac{a^{2}}{3 D_{\text {kerr,qu }}^{2}(r)}\right|}} .
\end{aligned}
$$

Both classical matchings as well as the one for the quantum Schwarzschild scenario monotonously increase and satisfy $D(r \rightarrow 0)=0$, as can be seen from the numerical results in Fig. 23. In contrast, the proper distance is identically zero in the quantum Kerr scenario, see (D2) Therefore, we only show the results for the Schwarzschild-AdS geometry in Fig. 21.

It turns out (cf. Sec. VIII) that the expression for the Hawking temperature in a quantum-improved spacetime contains terms proportional to the derivative of $D(r)$, hence using the above construction for the proper distance leads to diverging Hawking temperatures at all horizons. Therefore, in the following we also discuss the proper distance induced by the eigentime of a radially infalling observer, where this feature is absent.

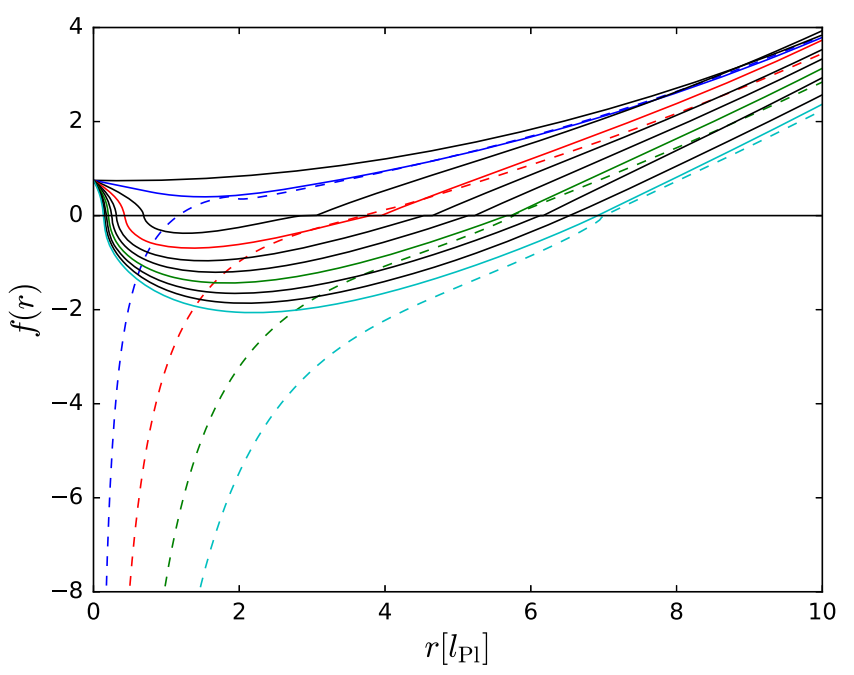

FIG. 21. $f(r)$ based on the radial path matching for increasing mass from top to bottom. Results, where $D(r)$ is computed consistently in a quantum-improved spacetime, are shown in solid, the dashed curves are the ones with a classically computed $D(r)$. With parameters $\Lambda_{0}=-0.1$ and $M=0.1,1,2,3,4,5,6$, $7,8,9,10 M_{\mathrm{Pl}}$. Curves of the same mass have the same color.

\section{b. Radial timelike geodesic}

The eigentime $\tau$ of an observer, initially at rest at $R$ and falling along a radial timelike geodesic into the singularity, can also be used to identify the momentum cutoff scale with a length scale by setting $D(r)=\tau(r)$. Derived in Appendix F, the eigentime for the Schwarzschild-(A)dS scenario reads

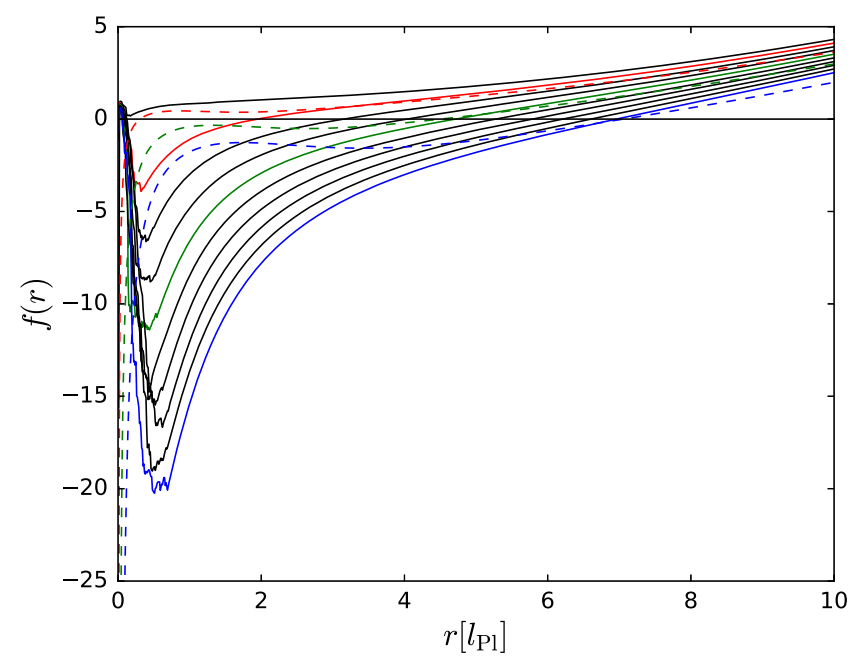

FIG. 22. $f(r)$ based on the radial geodesic matching for increasing mass from top to bottom. Results, where $D(r)$ is computed consistently in a quantum-improved spacetime, are shown in solid, the dashed curves are the ones with a classically computed $D(r)$. With parameters $\Lambda_{0}=-0.1$ and $M=$ $0.1,1,2,3,4,5,6,7,8,9,10 M_{\mathrm{Pl}}$. Curves of the same mass have the same color. 


$$
D(R)=\int_{0}^{R} \mathrm{~d} r \frac{1}{\sqrt{\left|E^{2}-f(r)\right|}}
$$

with $E=f(R)$ for an observer initially starting at rest. It is worth noting that for $E=0$, the integral reduces to the one in (C4). By fixing $E$, we equivalently specify the maximal distance $R$ of the observer from the origin. Independent on the particular value of $E$, the proper distance again exhibits poles if $E^{2}-f(r)=0$, now shifted by $E^{2}$ away from the horizons. Once more, (C7) gives rise to two different proper distances, referred to as either classical or quantum geodesic, depending on whether the underlying spacetime is based on the constant or running versions of $G$ and $\Lambda$. The lapse function for Schwarzschild-AdS based on the classical and quantum radial geodesic are displayed in Fig. 22.

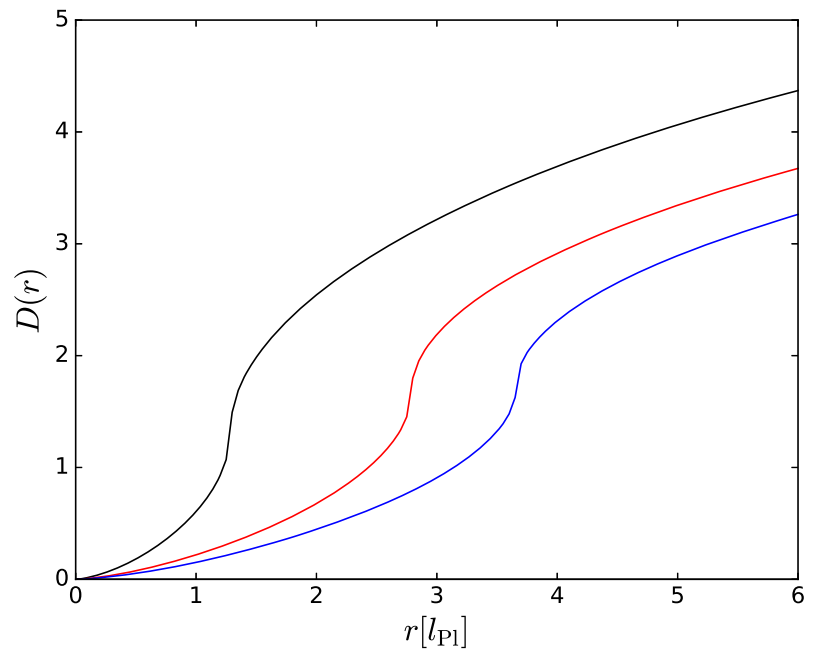

The analogous expression for the proper distance induced by an radial geodesic in the Kerr-(A)dS scenario reads (see Appendix G)

$$
\begin{aligned}
D(R) & =\int_{0}^{R} \mathrm{~d} r \frac{r^{2}}{\sqrt{\left|E^{2} \Xi^{2}\left[\left(r^{2}+a^{2}\right)^{2}-a^{2} \Delta_{r}\right]-r^{2} \Delta_{r}\right|}}, \\
E^{2} & =E^{2}(R)=\frac{R^{2} \Delta_{R}}{\Xi^{2}\left[\left(R^{2}+a^{2}\right)^{2}-a^{2} \Delta_{R}\right]},
\end{aligned}
$$

and reduces to $(\mathrm{C} 4)$ for $E=0$. Again, we achieved that there are no poles at the horizons. Once more, we have two versions depending on whether $G$ and $\Lambda$ are running or not. The numerical results can be found in Fig. 24; however, the proper distance in the quantum Kerr scenario is again identically zero.

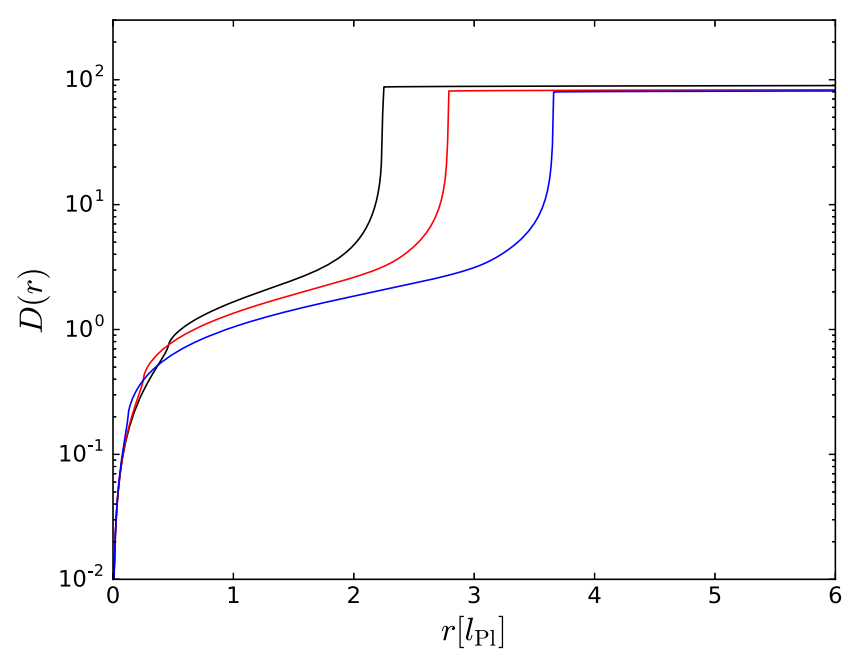

FIG. 23. Left: proper distance along a radial path through a classical Schwarzschild-AdS spacetime for three different masses $M=1,5,10 M_{\mathrm{Pl}}$. Right: the same for a quantum Schwarzschild-AdS spacetime.
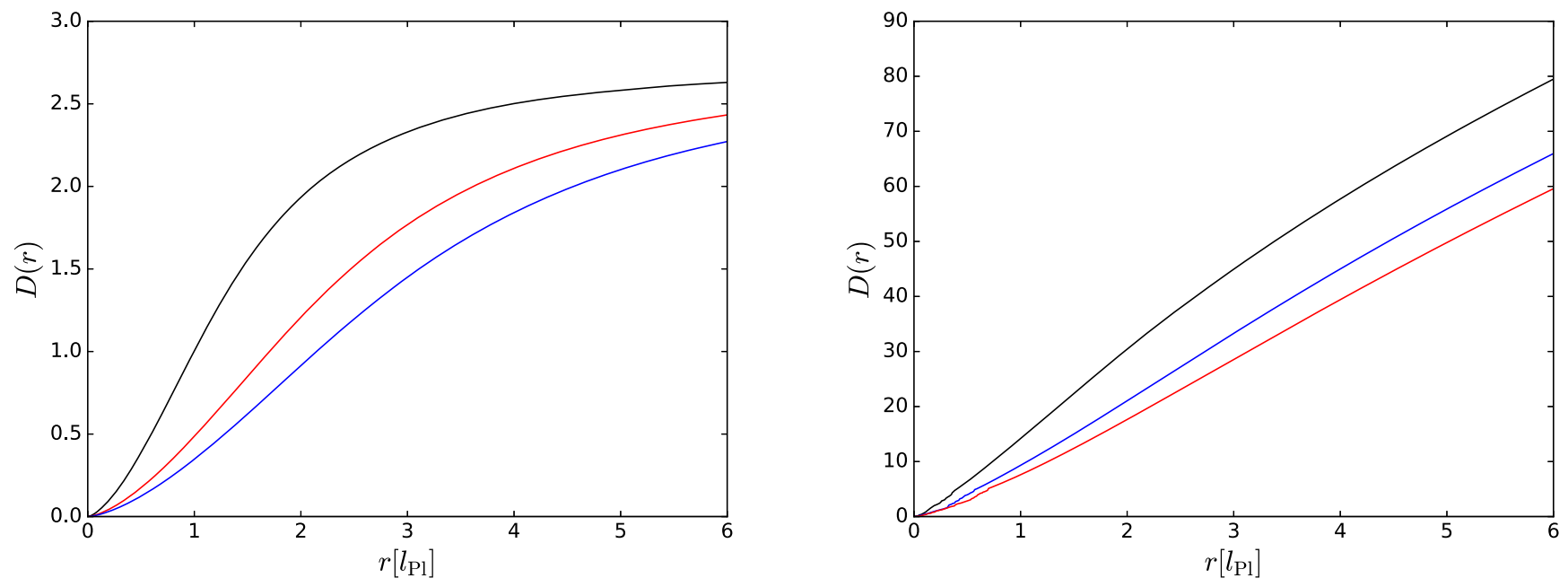

FIG. 24. Left: proper distance along a radial geodesic through a classical Schwarzschild-AdS spacetime for three different masses $M=1,5,10 M_{\mathrm{Pl}}$. Right: the same for a quantum Schwarzschild-AdS spacetime. 

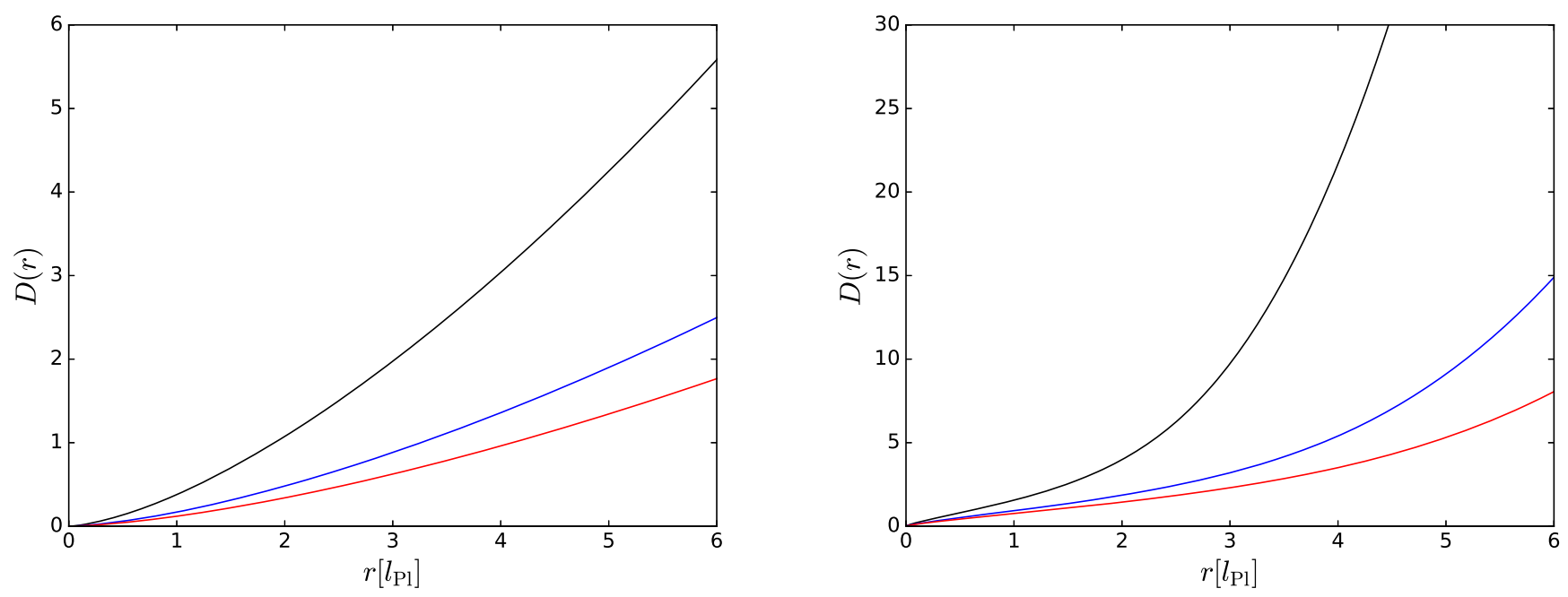

FIG. 25. Function $D(r)$ in classical Kretschmann matching (left) and for quantum Kretschmann scenario (right) for three different masses $M=1,5,10 M_{\mathrm{Pl}}$.

\section{APPENDIX D: SHAPE AND DIVERGENCES OF PROPER DISTANCES}

As can be seen from Fig. 23-25, all functions $D(r)$ are monotonously increasing, some proper distances display a rapid increase. In order to understand these jumps and possible divergences, we have to look at the integral expressions for each proper distance (C4), (C7), and (C8). The expression $h(r)$ under each square root can become zero, and if $h(r)$ has just a single root at $r=r_{0}<R$, the corresponding pole is integrable, causing a jump in the proper distance,

$$
\begin{aligned}
D(R) & =\int_{0}^{R} \mathrm{~d} r \frac{1}{\sqrt{h(r)}}=\int_{0}^{R} \mathrm{~d} r \frac{1}{\sqrt{\left(r-r_{0}\right) \tilde{h}(r)}} \\
& \sim \int_{0}^{R} \mathrm{~d} r\left(r-r_{0}\right)^{-1 / 2}
\end{aligned}
$$

where $\tilde{h}(r)$ has no root at $r=r_{0}$. However, once the multiplicity of $r_{0}$ is larger than one, the pole is not integrable anymore and $D(r)$ exhibits a divergence at $r=r_{0}$. In any case, $D^{\prime}(r)$ is diverging for the radial path proper distances, even at integrable poles of $D(r)$, as can be seen from (C6). In case of the classical radial path, the position of these poles has no direct physical significance; however, in the quantum case, the poles are located precisely at the horizons, because then, the function $h(r)$ is nothing other than the horizon condition. Thus, for extremal black holes when at least two horizons coincide, the quantum proper distance along a radial path is ill defined. $D^{\prime}(r)$ is always diverging at the horizons leading to a diverging Hawking temperature of the horizon, as is shown in Sec. VIII.

For this reason, we introduce the scenario with an infalling observer along a timelike, radial geodesic, in order to remove these problems, only due to the poor choice of the function $h(r)$ and absent in all other scenarios.
However, it turns out, that in both proper distance scenarios for Kerr-(A)dS with an underlying quantum spacetime, the proper distance must vanish identically, in order to satisfy the condition $D(0)=0$. For instance, this can be seen by solving (C6) in the limit $r \rightarrow 0$, satisfying the boundary condition $D(0)=\epsilon$, yielding

$$
D_{\mathrm{rad}, \mathrm{UV}}^{\mathrm{kerr}}(r)=\frac{\epsilon}{a^{\sqrt{3}}}\left(\sqrt{r^{2}+a^{2}}+r\right)^{\sqrt{3}} .
$$

Therefore, the solution vanishes identically in the limit $\epsilon \rightarrow 0$, which is confirmed also for the full, numerical solution of (C4). The same behavior is found for Kerr-(A) $\mathrm{dS}$, when the scale matching is based on the geodesic in a quantum-improved spacetime.

\section{APPENDIX E: UV LIMITS OF D(r)}

For statements about the curvature near the singularity and also for the construction of the Penrose diagrams, the UV-limit for each proper distance is needed.

The leading-order behavior in the UV for the classical proper distances, i.e., constant $G_{0}$ and $\Lambda_{0}$, can be obtained from (C4), (C7) and (C8) by approximating the integral in the limit $r \rightarrow 0$. For the identification based on the classical Kretschmann scalar (10), the UV behavior can easily be read off from (11).

In the quantum versions, the leading order of the proper distance in the UV-limit can be obtained by assuming a power law behavior of the form $D(r)=A r^{\alpha}$, with constants $A>0$ and $\alpha>0$ in order to satisfy the boundary condition $D(0)=0$. The constants $A$ and $\alpha$ can be determined by inserting this ansatz back into the above equations, now being an integral, differential or functional equation, respectively. All scenarios display monotonously increasing functions satisfying $D(0)=0$, apart from the quantum proper distance expressions for Kerr. They are 
identically zero, as an iterative algorithm for solving the integral equations shows.

For each scenario, the analytical UV-expression is listed in Table II. The numerical results for $D(r)$ are shown in Fig. 23-25. Furthermore, the leading-order exponent $\alpha$ can be extracted numerically from the slope of the linear relation between the proper distance $D(r)=A r^{\alpha}$ and its integral function $\mathcal{D}(r)=\frac{A}{\alpha+1} r^{\alpha+1}$ :

$$
\frac{\mathcal{D}(r)}{D(r)}=\frac{r}{\alpha+1} .
$$

This cross-check confirms agreement between numerical exponent and the one found analytically in Table II.

\section{APPENDIX F: EIGENTIME OF AN INFALLING OBSERVER IN A SCHWARZSCHILD-(A)DS GEOMETRY}

Another physically well-motivated choice for the integration path in $(\mathrm{C} 2)$ is the curve determined by an observer some distance away from the black hole, falling into the black hole along a radial timelike geodesic. Because the observer's four-velocity $u^{a}$ is conserved along geodesics, we normalize it to be

$$
-1 \stackrel{!}{=} u_{a} u^{a}
$$

Furthermore, we can choose the coordinate system such that the motion takes place only in the equatorial plane $\theta=\pi / 2$. Using (6), the normalization condition of the four-velocity in the equatorial plane reads:

$$
-f(r) \dot{t}^{2}+\frac{\dot{r}^{2}}{f(r)}+r^{2} \dot{\phi}^{2}=-1,
$$

where $(\dot{)}$ denotes the derivative with respect to the eigentime $\tau$. We have also conserved quantities $E$ and $L$ corresponding to the Killing vector fields $\xi^{a}=\left(\frac{\partial}{\partial t}\right)^{a}$ and $\psi^{a}=\left(\frac{\partial}{\partial \phi}\right)^{a}:$

$$
\begin{gathered}
E=-g_{a b} \xi^{a} u^{b}=f(r) \dot{t}, \\
L=g_{a b} \psi^{a} u^{b}=r^{2} \dot{\phi} .
\end{gathered}
$$

However, for simplicity, we will choose an observer with $L=0$. Inserting $E$ and $L$ back into (F2) to eliminate $\dot{t}$ and $\dot{\phi}$ leaves us with

$$
E^{2}=\dot{r}^{2}+f(r)
$$

This is a type of energy equation for the observer, at least in asymptotically flat spacetimes. We now have to specify the initial conditions for the observer. In the asymptotically flat spacetime, one usually places the observer initially at rest at $r=\infty$, still leaving $E$ finite. However, we cannot do that in the case of a nonvanishing cosmological constant, because $f(r)$ is diverging for $r \rightarrow \infty$. Therefore, we take rather an observer at rest $\left(\dot{r}^{2}=0\right)$ at some finite distance $R$ to determine $E$ :

$$
E^{2}=f(R)
$$

The proper distance is then given by the eigentime the observer needs to arrive at $r=0$ after starting at $R$, i.e., the integral over the eigentime along the geodesic:

$$
D(R)=\int_{0}^{R} \mathrm{~d} r \frac{1}{\sqrt{\left|E^{2}-f(r)\right|}}=\int_{0}^{R} \mathrm{~d} r \frac{1}{\sqrt{|f(R)-f(r)|}} .
$$

\section{APPENDIX G: EIGENTIME OF AN INFALLING OBSERVER IN A KERR-(A)DS GEOMETRY}

Following the same procedure for a timelike geodesic in the equatorial plane in Kerr-(A)dS, given by the metric (7), the normalization of the four-velocity is

$$
-1=g_{t t} \dot{t}^{2}+g_{\phi \phi} \dot{\phi}^{2}+2 g_{t \phi} \dot{t} \dot{\phi}+g_{r r} \dot{r}^{2},
$$

whereas the conserved quantities induced by the Killing vector fields $\xi^{a}=\left(\frac{\partial}{\partial t}\right)^{a}$ and $\psi^{a}=\left(\frac{\partial}{\partial \phi}\right)^{a}$ read

$$
\begin{gathered}
E=-g_{a b} \xi^{a} u^{b}=-g_{t t} \dot{t}-g_{t \phi} \dot{\phi}, \\
L=g_{a b} \psi^{a} u^{b}=g_{\phi \phi} \dot{\phi}+g_{t \phi} \dot{t} .
\end{gathered}
$$

Combining the equations and restricting again to $L=0$ yields the following radial equation,

$$
\dot{r}^{2}=\frac{E^{2} \Xi^{2}\left[\left(r^{2}+a^{2}\right)^{2}-a^{2} \Delta_{r}\right]-r^{2} \Delta_{r}}{r^{4}} .
$$

Subsequently, we arrive at the proper distance in a Kerr-(A) dS geometry induced by an infalling observer in the equatorial plane, initially starting at rest at $r=R$ and falling towards the singularity at $r=0$ :

$$
D(R)=\int_{0}^{R} \mathrm{~d} r \frac{r^{2}}{\sqrt{\left|E^{2} \Xi^{2}\left[\left(r^{2}+a^{2}\right)^{2}-a^{2} \Delta_{r}\right]-r^{2} \Delta_{r}\right|}},
$$

where $E$ is in this case then given by

$$
E^{2}=E^{2}(R)=\frac{R^{2} \Delta_{R}}{\Xi^{2}\left[\left(R^{2}+a^{2}\right)^{2}-a^{2} \Delta_{R}\right]} .
$$


[1] S. Weinberg, in General Relativity: An Einstein Centenary Survey, edited by S. W. Hawking and W. Israel (Cambridge University Press, Cambridge, England, 1979), p. 790.

[2] M. Reuter, Nonperturbative evolution equation for quantum gravity, Phys. Rev. D 57, 971 (1998).

[3] A. Bonanno and M. Reuter, Quantum gravity effects near the null black hole singularity, Phys. Rev. D 60, 084011 (1999).

[4] A. Bonanno and M. Reuter, Renormalization group improved black hole spacetimes, Phys. Rev. D 62, 043008 (2000).

[5] M. Reuter and E. Tuiran, Quantum gravity effects in the Kerr spacetime, Phys. Rev. D 83, 044041 (2011).

[6] B. Koch and F. Saueressig, Structural aspects of asymptotically safe black holes, Classical Quantum Gravity 31, 015006 (2014).

[7] K. Falls, D. F. Litim, and A. Raghuraman, Black holes and asymptotically safe gravity, Int. J. Mod. Phys. A 27, 1250019 (2012).

[8] B. Koch and F. Saueressig, Black holes within asymptotic safety, Int. J. Mod. Phys. A 29, 1430011 (2014).

[9] A. Bonanno, B. Koch, and A. Platania, Cosmic censorship in quantum Einstein gravity, Classical Quantum Gravity 34, 095012 (2017).

[10] A. Bonanno and M. Reuter, Spacetime structure of an evaporating black hole in quantum gravity, Phys. Rev. D 73, 083005 (2006).

[11] K. Falls and D. F. Litim, Black hole thermodynamics under the microscope, Phys. Rev. D 89, 084002 (2014).

[12] M. Reuter and H. Weyer, Running Newton constant, improved gravitational actions, and galaxy rotation curves, Phys. Rev. D 70, 124028 (2004).

[13] H. Emoto, Asymptotic safety of quantum gravity and improved spacetime of black hole singularity by cutoff identification, arXiv:hep-th/0511075.

[14] M. Niedermaier, The asymptotic safety scenario in quantum gravity: an introduction, Classical Quantum Gravity 24, R171 (2007).

[15] D. F. Litim, Renormalization group and the Planck scale, Phil. Trans. R. Soc. A 369, 2759 (2011).

[16] M. Reuter and F. Saueressig, Quantum Einstein gravity, New J. Phys. 14, 055022 (2012).

[17] A. Bonanno and F. Saueressig, Asymptotically safe cosmology - A status reportLa cosmologie asymptotiquement sûre: un rapport d'étape, C.R. Phys. 18, 254 (2017).
[18] R. Percacci, An Introduction to Covariant Quantum Gravity and Asymptotic Safety, 100 Years of General Relativity, Vol. 3 (World Scientific, Singapore, 2017).

[19] A. Eichhorn, Status of the Asymptotic Safety Paradigm for Quantum Gravity and Matter, Found. Phys. 48, 1407 (2018).

[20] T. Denz, J. M. Pawlowski, and M. Reichert, Towards apparent convergence in asymptotically safe quantum gravity, Eur. Phys. J. C 78, 336 (2018).

[21] W. Dittrich and M. Reuter, Effective Lagrangians in Quantum Electrodynamics (Springer Verlag, Berlin, 1985).

[22] G. W. Gibbons and S. W. Hawking, Cosmological event horizons, thermodynamics, and particle creation, Phys. Rev. D 15, 2738 (1977).

[23] M. Hindmarsh and I. D. Saltas, $f(R)$ gravity from the renormalization group, Phys. Rev. D 86, 064029 (2012).

[24] E. J. Copeland, C. Rahmede, and I. D. Saltas, Asymptotically safe Starobinsky inflation, Phys. Rev. D 91, 103530 (2015).

[25] B. Carter, Global structure of the Kerr family of gravitational fields, Phys. Rev. 174, 1559 (1968).

[26] E. Hackmann, C. Lammerzahl, V. Kagramanova, and J. Kunz, Analytical solution of the geodesic equation in Kerr(anti-) de Sitter space-times, Phys. Rev. D 81, 044020 (2010).

[27] M. Dafermos, Black holes without spacelike singularities, Commun. Math. Phys. 332, 729 (2014).

[28] L. Modesto, Disappearance of black hole singularity in quantum gravity, Phys. Rev. D 70, 124009 (2004).

[29] Y.-F. Cai and D. A. Easson, Asymptotically safe gravity as a scalar-tensor theory and its cosmological implications, Phys. Rev. D 84, 103502 (2011).

[30] M. Reuter and H. Weyer, Quantum gravity at astrophysical distances?, J. Cosmol. Astropart. Phys. 12 (2004) 001.

[31] B. Koch and I. Ramirez, Exact renormalization group with optimal scale and its application to cosmology, Classical Quantum Gravity 28, 055008 (2011).

[32] S. W. Hawking, Particle creation by black holes, Commun. Math. Phys. 43, 199 (1975).

[33] G. W. Gibbons and S. W. Hawking, Action integrals and partition functions in quantum gravity, Phys. Rev. D 15, 2752 (1977).

[34] S. Aretakis, Horizon instability of extremal black holes, Adv. Theor. Math. Phys. 19, 507 (2015).

[35] P. Chen, Y. C. Ong, and D.-h. Yeom, Black hole remnants and the information loss paradox, Phys. Rep. 603, 1 (2015). 